\title{
Review Article \\ Research Progress of Synthesis and Modification Methods Based on Dynamic Substructures
}

\author{
Jintao Su ${ }^{1}{ }^{1}$ and Bangdong Wang ${ }^{2}$ \\ ${ }^{1}$ State Key Laboratory of Mechanical Transmission, Chongqing University, Chongqing 400044, China \\ ${ }^{2}$ Saic-Iveco Hongyan Commercial Vehicle Co., Ltd., Chongqing 401122, China \\ Correspondence should be addressed to Jintao Su; nvh2012@163.com
}

Received 31 October 2019; Revised 20 January 2020; Accepted 6 February 2020; Published 9 March 2020

Academic Editor: F. Viadero

Copyright (c) 2020 Jintao Su and Bangdong Wang. This is an open access article distributed under the Creative Commons Attribution License, which permits unrestricted use, distribution, and reproduction in any medium, provided the original work is properly cited.

\begin{abstract}
In the process of model modification of large and complex structures, substructure synthesis method and modal reduction method have been widely used, but there are still some difficulties in precision control and engineering application in the process of model updating. In order to better study the dynamic response of the vibration substructure, the synthesis and correction method of the classical dynamic substructure is described in this paper, which provides a new idea for further engineering development. In the aspect of substructure synthesis method, the modal reduction of substructures, two methods of classical substructure synthesis, mechanical impedance method, singular value decomposition method, rigidity-flexibility equivalence, and transformation of degree of freedom are analyzed. The advantages and disadvantages of the above methods are discussed. In terms of substructure modification, the reference datum method, function dynamic modification method, neural network model modification, and frequency response function modification are analyzed, and the shortcomings of the dynamic substructure modification method are summarized. Finally, the development trend of dynamic substructure synthesis and modification algorithm is proposed.
\end{abstract}

\section{Introduction}

In the process of dynamic structure design and modeling analysis, the calculation of large complex structures requires a lot of computational cost and is not conducive to rapid modification and structural optimization in modern innovative design [1-3]. The vibration substructure synthesis method provides a solution to this kind of problem. It divides the overall structure into several interrelated substructures, and then according to the coordination conditions of displacement and force, the dynamic connection relationship between substructures and the whole structure is constructed, the dynamic characteristics of each substructure are calculated, and finally, the dynamic characteristics of the overall structure are determined according to the connection relationship.

Traditional dynamic substructure synthesis methods, such as fixed interface component mode synthesis method, coupled interface modal synthesis method, and mixed interface modal synthesis method, have higher-order modal truncation errors in modal synthesis, and there are some shortcomings in the accuracy and efficiency of substructure calculation. In recent years, dynamic frequency-domain substructures, including frequency response function (FRF) synthesis in frequency domain, noise reduction in frequency-domain transfer function, and dynamic frequency-domain substructure synthesis considering rotational degree of freedom, have developed rapidly in solving the problem of vibration subsystem synthesis, especially in the calculation of large-scale models. Frequency-domain dynamic substructures contain the FRF matrix of substructures and the modal information needed to solve them, which can calculate the dynamic characteristics of structures more accurately without the influence of higher-order modal truncation and has a good application prospect $[4,5]$.

In most cases, the results obtained by numerical analysis are not in good agreement with the experimental results. The 
reason is that connections insert the uncertainties in the system and that each separate system can be adequately described by the numerical model. When the error of the experimental results is large, the dynamic characteristics of the structural model obtained by the analysis will be quite different from the actual measurement results, even exceeds the design accuracy required in engineering practice. The case needs to correct the model, and the common methods of model modification include matrix modification, parametric modification, genetic algorithm (GA), simulated annealing (SA) modification, and neural network modification. The purpose of structural model modification is to minimize the error between the theory model and the actual structure.

This paper reviews the synthesis and modification of substructures. In terms of substructure synthesis, modal reduction method, singular value decomposition method of frequency response function, and principle and application of angular degree-of-freedom are analyzed. In terms of substructure modification, the dynamic method of objective function, parametric correction, neural network correction, and sensitivity correction are analyzed. Finally, the research trends of substructure synthesis and modification methods are proposed.

\section{Dynamic Substructure}

2.1. Dynamic Substructure Synthesis. The internal relationship and connection interface of the substructure are shown in Figures 1 and 2. The dynamic substructure method is a comprehensive method that divides the whole structure into various interrelated substructures and then connects each substructure according to the coordination equation of displacement and force. Substructure physical domain is divided into eigenvalue analysis and the Fourier transform, which are decomposed into time-domain modal and frequency-domain, respectively, which are transformed by modal superposition and modal recognition. The main research methods of dynamic substructure are modal synthesis method, frequency-domain synthesis method, and timedomain synthesis method. Among them, the component mode synthesis (CMS) method is a commonly used method for computing dynamic subsystems. Klerk et al. [6], Voormeeren et al. [7], and Craig [8] have reviewed the fixed boundary, free boundary, mixed boundary, and load boundary methods, respectively. In the theoretical research of dynamic subsystems, Hou [9] proposed a free-interface mode synthesis method, which uses low-order primary modes and ignores the influence of the remaining higherorder modes. Craig and Chang [10] improved the HOU method, introduced the residual modes into the dynamic subsystem, and improved the accuracy and convergence of the free interface synthesis method. Suarez and Singh [11] improved the modal truncation method based on the highorder modal superposition method and the fixed interface principal mode and increased the error estimation of eigenvalues. Bishop and Johnson [12] improved the methods of mechanical impedance and admittance and applied them to the substructure analysis of simple structural beam

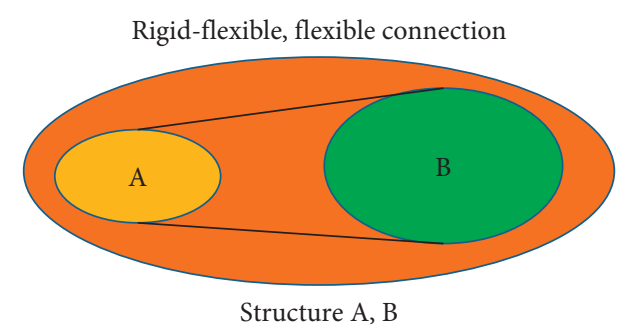

Figure 1: The internal relations of dynamic substructures.

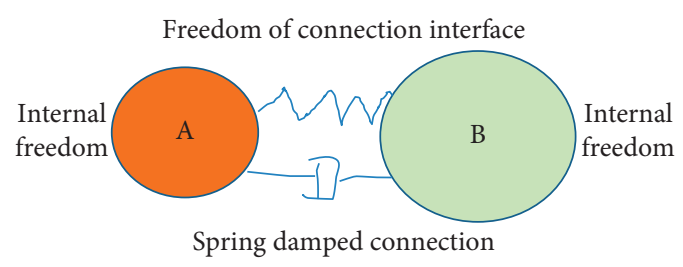

FIgURE 2: The connection interface of dynamic substructure.

elements. O’Hara [13] innovated and perfected mechanical impedance and mechanical admittance amplification based on BISHOP and JOHNSON and introduced the method into the multidegree-of-freedom complex mechanical structures. Gordis [14] first proposed the impulse-based substructure method (IBS) using impulse response function (IRF) to connect the substructure. The IBS is an inverse Fourier transformation of the Lagrange multiplier frequency-based substructure method. Therefore, IBS has the same advantage that lowers errors compared with component mode synthesis. However, it is better in dealing with transient impact problems. Rixen and van der Valk [15] first achieved successful work using the IBS method in 2010 with rigidly connection interface. In the paper by Dong et al. [16], a new method was applied to a complex spacecraft which have dampers used to isolate vibrations; specifically, apart from rigid connections, elastic connections are also took into account, which was called the rigid-elastic hybrid joint description method. In the case, the soft landing of a legged lunar lander with rigid and elastic joints was modeled and processed via the proposed method and is more accurate and less time costing compared to component mode synthesis since IRFs include high frequency.

2.2. Model Reduction of Dynamic Substructure. For complex large-scale dynamic finite element models, the calculation accuracy and scale are the key to solve such problems. Using model condensation or substructure method for dynamic analysis, or mixing with experimental data to establish accurate models, can effectively reduce the amount of calculation. Common model condensation methods include GUYA condensation [17], Improved Reduction System (IRS) method [18], dynamic condensation method [19], and System Equivalent Reduction Expansion Process (SEREP) method [20]. Based on multilevel condensation, Raynaud et al. [21] proposed a method to modify the complex model. It is a new method based on compression model and frequency response. Firstly, the large-scale complex model is 
reduced to several hundred degrees of freedom, and then the model is compressed to the measurement point, which effectively solves the problem of superlarge-scale computation. A new reduction method called Gram-Schmidt interface (GSI) gives an approach to solve the problems that the classical component mode synthesis method still has so many interface dofs which need decrement. Tran [22] demonstrates that the GSI method is efficient especially for cyclic symmetric structures. Battiato [23] applied it to a turbomachinery consisting of a blade coupled with a sector of disk which was treated as cyclic symmetry conditions. In particular, in terms of each substructure, the classical Craig-Bampton method was first used; then, the SGI was applied to further interface mode reduction. Frank [24] presented a new model order reduction approach, multilevel substructuring (MLS). The basic idea is to first decompose a structure to several smaller substructures; then, each substructure will be decomposed to a smaller substructure until enough small substructures are obtained. Naturally, according to the level, it could be categorized to single-level case, two-level case, and so on. Then, in terms of each substructure, many different model reduction methods could be used. In the case, a SVD-based method, balanced truncation, was chosen. Finally, it demonstrates the method is a promising tool for the reduction of very large structures when the direct application of reduction methods is not possible.

2.3. Application of Dynamic Substructure. In the application of the dynamic subsystem method, Sung [25] used the mixed interface modal synthesis method to model the acousticvibration system and predicted the internal noise response caused by random road excitation. Lee et al. [26] established vehicle model of body, suspension, tire, and other subsystems using the subsystem modal synthesis method and calculated vehicle modal and vibration response after subsystem synthesis. Qiu et al. [27] adopted the displacement expansion terms of mixed modes to derive the accurate substructure dynamic mixing function method and applied it to the dynamic analysis to correct the theory of mixed interface. Donders et al. [28] decompose the whole structure into two independent substructures, used the wave theory to reconfigure the decomposed substructures, and used the reduction technology to solve and calculate the body model. The wave reduction technology combines and decomposes the whole structure and then combines the degree of freedom of the connecting interface to solve the dynamic characteristics of the structure. Although the modal synthesis method, condensation method, and mechanical impedance admittance method have been popularized and applied in some engineering and technical fields, this method has some limitations and is not applicable to superlarge complex structures, complex boundary conditions, and uncertain boundary conditions. With the progress of science and technology and the development of testing technology, the uncertainties are increasingly solved by testing technology. On the contrary, with the increasing accuracy of frequency response function measurement, the application of FRF direct measurement results in frequencydomain dynamic substructure synthesis is possible. Ewins [29] summarized and elaborated the measurement and synthesis methods of dynamic substructure frequency response function, which played a certain role in the direct application of frequency response function. An improved FRF-based substructure synthesis by Liu [30] was applied to vehicle axle noise analysis. In the application, the body was replaced by FRFs at the attachments with the chassis and the chassis was modeled by the finite element method. Later, the dominant transfer paths were identified through the power flows calculated by the method. Holzwarth and Eberhard [31] achieved an application to elastic multibody systems (EMBS) with SVD-based component mode synthesis, CMSGram method. Elastic multibody systems (EMBS) generally consist of several elastic bodies and joints, which were used more and more in various products such as acoustic analyses where the deformation could not be neglected. Generally, the elastic bodies are modeled with finite element analysis; hence, the reduction of the dofs of elastic bodies is naturally the first step needed to solve. In the case, the model consists of an elastic body and an elastic driver's seat which separately have 2 million and 0.15 million dofs. While the model is reduced to dofs 280 (body) and 100 (seat), the CMS-Gram method captures all the eigenmodes precisely in the frequency band $(0-60 \mathrm{~Hz})$.

\section{Frequency Response Function Substructure}

3.1. Frequency Response Matrix Substructure. The substructure method based on FRF is to synthesize the FRF matrices between different substructures directly to build the model of multiple subsystems. The original FRF substructure is simple, but there are two inverse processes of matrix [12]:

(1) Jetmundsen et al. [32] summarized the frequency response function substructure method and deduced the condition of displacement and force balance between substructures. This method greatly improved the mechanical admittance and impedance method. It does not need the inversion of the frequency response matrix of the whole structure, but only the inversion of the frequency response function matrix at the junction. The operation greatly reduces the difficulty of solving calculation and the order of matrix solution. The substructures studied in this method are shown in Figures 3 and 4. According to the relationship between the separation and combination of substructures and the distribution of frequency response matrix, the frequency response matrix of the summary structure is obtained. The rigid connection of the degree of freedom $b$ and $d$ of the connection interface does not consider the flexible treatment of the connection interface 


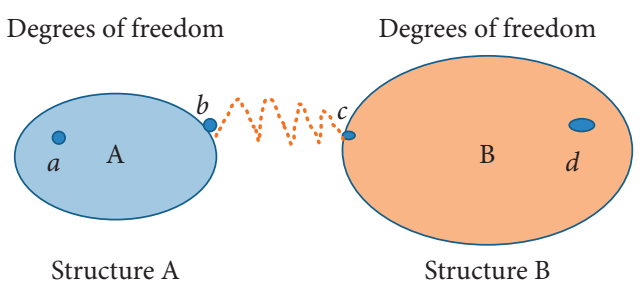

Figure 3: Dynamic substructure separation state.

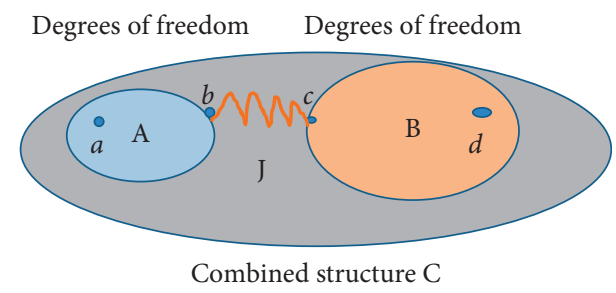

Figure 4: Dynamic substructure combined state.

$$
\begin{aligned}
H^{A} & =\left[\begin{array}{ll}
H_{\bar{a} \bar{a}}^{A} & H_{\bar{a} \bar{b}}^{A} \\
H_{\bar{b} \bar{a}}^{A} & H_{\bar{b} \bar{b}}^{A}
\end{array}\right], \\
H^{B} & =\left[\begin{array}{ll}
H_{\bar{c} \bar{c}}^{B} & H_{\bar{c} \bar{d}}^{B} \\
H_{\bar{d} \bar{c}}^{B} & H_{\bar{d} \bar{d}}^{B}
\end{array}\right] .
\end{aligned}
$$

According to the displacement force coordinate equilibrium equation,

$$
x_{\bar{b}}^{A}=x \frac{B}{d} \stackrel{\text { def }}{=} x_{j}^{C} .
$$

The combined structure frequency response function matrix is obtained:

$$
\begin{aligned}
H^{c}= & {\left[\begin{array}{lll}
H_{a a}^{c} & H_{a j}^{c} & H_{a c}^{c} \\
H_{j a}^{c} & H_{j j}^{c} & H_{j c}^{c} \\
H_{c a}^{c} & H_{c j}^{c} & H_{c c}^{c}
\end{array}\right]=\left[\begin{array}{ccc}
H_{a a}^{A} & H_{a b}^{A} & 0 \\
H_{b a}^{A} & H_{b b}^{A} & 0 \\
0 & 0 & H_{c c}^{B}
\end{array}\right] } \\
& -\left[\begin{array}{c}
H_{a b}^{A} \\
H_{b b}^{A} \\
-H_{c d}^{B}
\end{array}\right]\left(H_{b b}^{A}+H_{a a}^{B}\right)^{-1}\left[\begin{array}{c}
H_{a b}^{A} \\
H_{b b}^{A} \\
-H_{c d}^{B}
\end{array}\right]^{T} .
\end{aligned}
$$

(2) Ren and Beards [33] made a great improvement on the dynamic substructure method based on Jetmundsen and proposed a calculation method suitable for the synthesis of multiple substructures, and some preliminary calculations and applications can be carried out in coupled nonindependent substructures.

(3) Another frequency-domain substructure method is the mechanical admittance and impedance substructure synthesis method which takes into account the connection components and connection characteristics. This method integrates the characteristics of the previous method and considers the impedance and admittance of the connected components in the substructure synthesis. The mechanical admittance matrix and the connected mechanical impedance matrix of the original structure are

$$
\begin{aligned}
& {\left[\begin{array}{l}
x_{\bar{a}} \\
x_{\bar{b}} \\
x_{\bar{c}}
\end{array}\right]=\left[\begin{array}{lll}
H_{\bar{a} \bar{a}} & H_{\bar{a} \bar{b}} & H_{\bar{a} \bar{c}} \\
H_{\bar{b} \bar{a}} & H_{\bar{b} \bar{b}} & H_{\bar{b} \bar{c}} \\
H_{\bar{c} \bar{a}} & H_{\bar{c} \bar{b}} & H_{\bar{c} \bar{c}}
\end{array}\right]\left[\begin{array}{l}
f_{\bar{a}} \\
f_{\bar{b}} \\
f_{\bar{c}}
\end{array}\right],} \\
& {\left[\begin{array}{l}
f_{\widehat{b}} \\
f_{\widehat{c}}
\end{array}\right]=\left[\begin{array}{ll}
Z_{\widehat{b} \widehat{b}} & Z_{\widehat{b} \bar{c}} \\
Z_{\widehat{c} \hat{b}} & Z_{\widehat{c} \widehat{c}}
\end{array}\right]\left[\begin{array}{l}
x_{\widehat{b}} \\
x_{\widehat{c}}
\end{array}\right],}
\end{aligned}
$$

where $\vec{a}, \vec{b}$, and $\vec{c}$ represent the internal degrees of freedom of the original composite structure, $\hat{b}$ and $\widehat{c}$ are the connecting degrees of freedom of the connecting parts, $b$ and $c$ denote the interface degrees of freedom of the combination, and $a, b$, and $c$ are the postcombination degrees of freedom.

According to the displacement and force coordination equation of the mechanical impedance admittance substructure,

$$
\begin{gathered}
{\left[\begin{array}{l}
x_{\bar{b}} \\
x_{c}
\end{array}\right]=\left[\begin{array}{l}
x_{\widehat{b}} \\
x_{\widehat{c}}
\end{array}\right] \stackrel{\text { def }}{=}\left[\begin{array}{l}
x_{b} \\
x_{c}
\end{array}\right],} \\
x_{\bar{a}} \stackrel{\text { def }}{=} x_{a}, \\
{\left[\begin{array}{l}
f_{\bar{b}} \\
f_{\bar{c}}
\end{array}\right]+\left[\begin{array}{c}
f_{\widehat{b}} \\
f_{\widehat{c}}
\end{array}\right]=\left[\begin{array}{l}
f_{b} \\
f_{c}
\end{array}\right],} \\
x_{\bar{a}} \stackrel{\text { def }}{=} x_{a} .
\end{gathered}
$$

According to equations (5) and (6) deduced,

$$
\begin{aligned}
& \left(Z_{\widehat{b} \widehat{b}} H_{\bar{b} \bar{a}}+Z_{\widehat{b} \widehat{c}} H_{\bar{c} \bar{a}}\right) f_{\bar{a}}+\left(Z_{\widehat{b} \widehat{b}} H_{\bar{b} \bar{b}}+Z_{\widehat{b}} H_{\bar{c} \bar{b}}\right) f_{\bar{b}} \\
& +\left(Z_{\widehat{b} \bar{b}} H_{\bar{b} \bar{c}}+Z_{\widehat{b} \widehat{c}} H_{\bar{c} \bar{c}}\right) f_{\bar{c}}=f_{\widehat{b}}, \\
& \left(Z_{\widehat{c} \widehat{b}} H_{\bar{b} \bar{a}}+Z_{\widehat{c} \widehat{c}} H_{\bar{c} \bar{a}}\right) f_{\bar{a}}+\left(Z_{\widehat{c} \widehat{b}} H_{\bar{b} \bar{b}}+Z_{\widehat{c} \widehat{c}} H_{\bar{c} \bar{b}}\right) f_{\bar{b}} \\
& \quad+\left(Z_{\widehat{c} \widehat{b}} H_{\bar{b} \bar{c}}+Z_{\widehat{c} \widehat{c}} H_{\bar{c} \bar{c}}\right) f_{\bar{c}}=f_{\widehat{c}} .
\end{aligned}
$$

Substitute equation (7) into equation (8), and derive

$$
\begin{aligned}
{\left[\begin{array}{c}
f_{\widehat{b}} \\
f_{\widehat{c}}
\end{array}\right] } & =\Delta^{-1}\left[\begin{array}{ll}
Z_{\widehat{b} \widehat{b}} & Z_{\widehat{b} \widehat{c}} \\
Z_{\widehat{c} \widehat{b}} & Z_{\widehat{c} \widehat{c}}
\end{array}\right]\left[\begin{array}{lll}
H_{\bar{b} \bar{a}} & H_{\bar{b} \bar{b}} & H_{\bar{b} \bar{c}} \\
H_{\bar{c} \bar{a}} & H_{\bar{c} \bar{b}} & H_{\bar{c} \bar{c}}
\end{array}\right]\left[\begin{array}{c}
f_{a} \\
f_{b} \\
f_{c}
\end{array}\right], \\
\Delta_{1} & =I+\left[\begin{array}{ll}
Z_{\widehat{b} \widehat{b}} & Z_{\widehat{b} \widehat{c}} \\
Z_{\widehat{c} \widehat{b}} & Z_{\widehat{c} \widehat{c}}
\end{array}\right]\left[\begin{array}{ll}
H_{\bar{b} \bar{b}} & H_{\bar{b} \bar{c}} \\
H_{\bar{c} \bar{b}} & H_{\bar{c} \bar{c}}
\end{array}\right] .
\end{aligned}
$$

The frequency response matrix of the whole structure is 


$$
\begin{aligned}
& {\left[\begin{array}{lll}
H_{a a} & H_{a b} & H_{a c} \\
H_{b a} & H_{b b} & H_{b c} \\
H_{c a} & H_{c b} & H_{c c}
\end{array}\right]=\left[\begin{array}{lll}
H_{\bar{a} a} & H_{\bar{a} \bar{b}} & H_{\bar{a}_{\bar{c}}} \\
H_{\bar{b} \bar{a}} & H_{\bar{b} \bar{b}} & H_{\bar{b} \bar{c}} \\
H_{\overline{c a}} & H_{\bar{c} \bar{b}} & H_{\bar{c} \bar{c}}
\end{array}\right]} \\
& -\left[\begin{array}{ll}
H_{\bar{a} \bar{b}} & H_{\overline{a_{\bar{c}}}} \\
H_{\bar{b} \bar{b}} & H_{\bar{b} \bar{c}} \\
H_{\bar{c} \bar{b}} & H_{\bar{c} \bar{c}}
\end{array}\right] \Delta_{1}^{-1}\left[\begin{array}{ll}
Z_{\widehat{b} \bar{b}} & Z_{\widehat{b} \widehat{c}} \\
Z_{\widehat{c} \bar{b}} & Z_{\bar{c} \widehat{c}}
\end{array}\right]\left[\begin{array}{ll}
H_{\bar{a} \bar{b}} & H_{\bar{a}_{\bar{c}}} \\
H_{\bar{b} \bar{b}} & H_{\bar{b} \bar{c}} \\
H_{\bar{c} \bar{b}} & H_{\overline{c c}}
\end{array}\right]^{T} .
\end{aligned}
$$

In the process of solving mechanical impedance and admittance of dynamic subsystem, it is necessary to inverse the frequency response function matrix of the whole subsystem matrix. However, the frequency response matrix tested by each substructure has ill-conditioned matrix in the resonance region, and there is a certain theoretical error in the inverse operation of ill-conditioned matrix, thus affecting the fitting effect and operation accuracy of the whole multisystem. Imregun and Robb [34] and Ewins [29] proposed that the modal of substructure can be inversely fitted according to the test frequency response function of simple results, and the frequency response function can be calculated according to the modal inverse operation of simple substructure, which can improve the fitting accuracy between substructure of separation state and substructure of combined state. However, this method is only suitable for substructures with simple degrees of freedom.

3.2. Singular Value Decomposition Method for Noise Reduction. In the subspace noise reduction method based on singular value decomposition, the Fourier transform is first used to transform the frequency response function into the pulse response function in the time domain. For the frequency response function with noise, the inverse discrete Fourier transform is

$$
h_{k}=\frac{1}{n} \sum_{s=0}^{n-1} H_{s} e^{j(2 \pi s k / n)}, \quad k=0,1, \ldots, n-1,
$$

where $h_{k}$ is the impulse frequency response function, $H$ and $h$ are discrete observation sequences, and $n$ is the length of the response impulse sequence. For the one-dimensional time series, an $m$-dimensional phase space can be reconstructed so that the reconstructed phase space has the same dynamic characteristics as the studied system. Assuming that the embedding dimension is $m$ and the delay time is $\tau$, the phase space matrix of the impulse response function is reconstructed by using the delay coordinate method, and the reconstructed phase space matrix $Y$ is

$$
Y=\left[\begin{array}{c}
y_{1+(m-1) \tau} \\
y_{2+(m-1) \tau} \\
\vdots \\
y_{n}
\end{array}\right]=\left[\begin{array}{cccc}
h_{1+(m-1) \tau} & \cdots & h_{1+\tau} & h_{1} \\
h_{2+(m-1) \tau} & \cdots & h_{2+\tau} & h_{2} \\
\vdots & \cdots & \ddots & \vdots \\
h_{n} & \cdots & h_{n-(m-2) \tau} & h_{n-(m-1) \tau}
\end{array}\right] .
$$

For noise reduction analysis, set extended time $\tau=1$, and reconstructed phase space matrix $Y$ can be expressed as

$$
Y=\left[\begin{array}{cccc}
h_{m} & \cdots & h_{2} & h_{1} \\
h_{1+m} & \cdots & h_{3} & h_{2} \\
\vdots & \cdots & \ddots & \vdots \\
h_{n} & \cdots & h_{n-m+2} & h_{n-m+1}
\end{array}\right] .
$$

Assuming that there is additive noise in the frequency response function, the phase space matrix $Y$ can be written as

$$
Y=X+N
$$

where $Y, X$, and $N$, respectively, represent the reconstruction matrix of the surface impulse response function of the original signal with noise, pure signal, and noise signal, and the above equation is singular value decomposition, i.e.,

$$
Y=U \sum V^{T}=\left[U_{y 1} U_{y 2}\right]\left[\begin{array}{cc}
\sum_{y 1} & 0 \\
0 & \sum_{y 2}
\end{array}\right]\left[\begin{array}{c}
V_{y 1}^{T} \\
V_{y 2}^{T}
\end{array}\right]
$$

where matrix $U$ and matrix $V$ are the left and right singular matrices of matrix $Y$, respectively, and are orthogonal matrices and $\sum$ is the singular value matrix.

Assuming the rank of the pure signal $X$ is $r$, the singular value decomposition of $X$ is expressed as

$$
X=U_{x} \sum_{x} V_{x}^{T}=\left[U_{x 1} U_{x 2}\right]\left[\begin{array}{cc}
\sum_{x 1} & 0 \\
0 & 0
\end{array}\right]\left[\begin{array}{c}
V_{x 1}^{T} \\
V_{x 2}^{T}
\end{array}\right] .
$$

It is further assumed that the noise quotation marks are Gaussian white noise signals, whose mean is zero and variance is $\sigma_{n}$, and the noise signal is not correlated with the pure signal $X$; then,

$$
\begin{aligned}
N^{T} N & =\sigma_{n} E, \\
X^{T} N & =0,
\end{aligned}
$$

where $E$ is the identity matrix and the phase space matrix $Y$ satisfying the above conditions is expressed as

$$
\begin{aligned}
Y= & X+N \\
= & U_{x 1} \sum_{x 1} V_{x 1}^{T}+N\left(V_{x 1} V_{x 1}^{T}+V_{x 2} V_{x 2}^{T}\right) \\
= & {\left[\left(U_{x 1} \sum_{x 1}+N V_{x 1}^{T}\right)\left(\sum_{x 1}^{2}+\sigma_{n}^{2} E_{r}\right)^{-1 / 2} N V_{x 2} \sigma_{n}^{-1}\right] \times } \\
& \cdot\left[\begin{array}{cc}
\sqrt{\sum_{x 1}^{2}+\sigma_{n}^{2} E_{r}} & 0 \\
0 & \sigma_{r} E_{m-r}
\end{array}\right]\left[\begin{array}{c}
V_{x 1}^{T} \\
V_{x 2}^{T}
\end{array}\right] \\
= & {\left[\begin{array}{ll}
U_{y 1} & U_{y 2}
\end{array}\right]\left[\begin{array}{cc}
\sum_{y 1} & 0 \\
0 & \sum_{y 2}
\end{array}\right]\left[\begin{array}{c}
V_{y 1}^{T} \\
V_{y 2}^{T}
\end{array}\right], }
\end{aligned}
$$

where $\sum_{y 1}=\sqrt{\sum_{x 1}^{2}+\sigma_{n}^{2} E_{r}}, \sum_{y 2}=\sigma_{n} E_{m-r}, \sum_{x 1}$ is the nonzero singular value of the pure signal, and $E$ is the single matrix. The above formula expresses the relationship between the reconstructed phase space matrix $Y$ of the impulse response function with noise and the singular value of the reconstructed phase space matrix $X$ of the pure impulse response function. In the least square sense, the optimal estimation of the reconstructed phase space matrix $X$ of the pure impulse response needs to be satisfied: 


$$
\min _{x}=\|\widehat{X}-X\|_{F}^{2}
$$

where \|\|$_{F}$ is the Frobenius norm and the least-squares estimation of the reconstructed phase space matrix $X$ of the pure impulse response function is obtained by solving the above equation:

$$
\widehat{X}_{L S}=U_{y 1} \sum_{y 1} V_{y 1}^{T}=\sum_{k=1}^{r} \sigma_{k} U_{k} V_{k}^{T},
$$

where $U_{k}$ and $V_{k}$ are left and right singular matrix vectors and $\sum_{y 1}$ is diagonal matrix. If its diagonal element $\sigma_{1} \sigma_{2} \ldots \sigma_{r}$ satisfies $\sigma_{1}>\sigma_{2}>\cdots>\sigma_{r}, \widehat{X}_{L S}$ is considered to be the approximation to $Y$.

Another estimate of the minimum variance of the pure impulse response $X$ is to find the linear filter $L$ of $Y$, which satisfies

$$
\min _{L}=\|Y L-X\|_{F}^{2}
$$

Solve the above equation to get the optimal matrix $L$, then the optimal estimate of $X$ is

$$
\widehat{X}_{M V}=U_{y 1} \sum_{M V} V_{y 1}^{T}=\sum_{k=1}^{r} \frac{\sigma_{k}^{2}-\sigma_{n}^{2}}{\sigma_{k}} U_{k} V_{k}^{T} .
$$

The impulse response function sequence after denoising is obtained by the following equation:

$$
h^{\prime}=\frac{1}{k-l+1} \sum_{k=1}^{r} \widehat{X}_{i-j+1, m+1-j},
$$

where $l=\max (1, i-n+m)$ and $k=\max (m, i)$, after obtaining the impulse response function sequence $h^{\prime}$ after denoising, and the frequency response function after denoising can be obtained through the inverse Fourier transform.

\subsection{Application of Frequency Response Function Noise} Reduction. In the synthesis of substructure frequency response function, inverse operation is required for the frequency response matrix of interface measurement points, which is very sensitive to signal noise. In view of the noise problems existing in the excitation and response of the actual measurement of intermediate frequency response function, $\mathrm{H} 1, \mathrm{H} 2, \mathrm{H} 3, \mathrm{H} 4, \mathrm{HV}$, and other classical methods are generally adopted in the project. This method is to estimate the frequency response function through the cross-spectrum average method of test signals before obtaining the frequency response function after completing the excitation and response measurement, so as to obtain the frequency response function with high SNR. However, the classical method is usually used to estimate the frequency response function with residual noise. Sanliturk and Cakar [35] used the singular value decomposition (SVD) method to test the noise reduction processing to obtain the frequency response function. Its denoising idea is to reconstruct the phase space of the frequency response function with noise. The reconstructed phase space matrix is decomposed into signal subspace and noise signal subspace by the singular value decomposition method. The inverse transform of singular value decomposition is used to estimate the matrix of signal subspace, and the inverse process of phase space reconstruction is used to obtain the frequency response function after noise reduction. Ephraim and Van Trees [36] decompose noise signal into subspace by eigenvalue decomposition of covariance matrix. Signal error is divided into signal distortion error and residual noise. Two evaluation criteria, TDC and SDC, are used for optimal linear estimation and compared with frequency algorithm. It proves its advantages in noise reduction. Johnson and Povinelli [37] applied the TDC method of subspace denoising algorithm to speech test signal denoising and achieved good denoising effect. The difference between TDC algorithm and SDC algorithm was compared, and the two factors of signal distortion error and residual noise were fully considered. $\mathrm{Hu}$ and Loizou [38] conducted covariance and diagonalization of test signal noise signal and extended Ephraim and Van Trees [36] method to signal noise reduction. Hermus et al. [39] summarized subspace noise reduction method and pointed out that, compared with spectral subtraction, automatic noise reduction method reduced residual noise and improved the accuracy of identification.

\subsection{Considering the Equivalence of Rigidity and Flexibility with Rotational Degree of Freedom}

(1) According to an equivalent multipoint connection (EMPC) method proposed by Klerk and Rixen et al., the frequency response equation of substructure $a$ is

$$
\left[\begin{array}{l}
u_{i} \\
u_{c}
\end{array}\right]^{a}=\left[\begin{array}{ll}
H_{i i} & H_{i c} \\
H_{c i} & H_{c c}
\end{array}\right]^{a}\left[\begin{array}{l}
f_{i} \\
f_{c}
\end{array}\right]^{a},
$$

where subscripts $i$ and $c$, respectively, represent the internal and interfacial degrees of freedom of the substructure, and the complete structural interface information includes all frequency response functions of the translational and rotational degrees of freedom of the node, so $C$ contains translational and rotational degrees of freedom information. $u=\left[\begin{array}{ll}u_{i} & u_{c}\end{array}\right]^{T}$ is defined as the response matrix of internal degrees of freedom and interface degrees of freedom of substructure, $f$ is the force element, the transformation matrix is defined as $R$, and the interface degrees $u_{c}$ of freedom of substructure $a$ can be expressed as

$$
u_{c}=R u_{\mathrm{ma}}+\mu
$$

where the equivalent principal degree of freedom response matrix $u_{\mathrm{mal}}^{(0)}$ contains three rotational degrees of freedom information of the interface and the transformation matrix $R_{1}$ is

$$
R_{1}=\left[\begin{array}{cc}
E & -L_{1} \\
\vdots & \vdots \\
E & -L_{n}
\end{array}\right] \text {. }
$$


$L_{i}=\left[\begin{array}{ccc}0 & -z_{i} & y_{i} \\ z_{i} & 0 & -x_{i} \\ -z_{i} & -x_{i} & 0\end{array}\right]$, an antisymmetric matrix, is the coordinate matrix of any measurement point $i$ on the interface in the substructure coordinate system.

$R_{1}$ shows the modal mode matrix of the interface rigid body motion, and the integrated transformation matrix of the internal degrees of freedom and the interface degrees of freedom of the joint substructure can be expressed as

$$
T_{r 1}=\left[\begin{array}{cc}
E & 0 \\
0 & R_{1}
\end{array}\right]
$$

According to the above formula, the frequency response function of $u_{\mathrm{mal}}^{(0)}$ with equivalent degrees of freedom of interface rigidity can be expressed as $\widetilde{H}=T_{r 1}^{+} H_{r 1}^{+T}$, where $T_{r 1}^{T}$ is the Moore-Penrose inverse matrix.

(2) The equivalent degree of freedom of interface flexibility is a special case of rigid equivalent degree of freedom. Firstly, the degree of freedom of measurement point of interface $u_{c}$ should be determined. According to the EMPC method, the connection interface is regarded as a whole rigid surface, and the equivalent degree of freedom $u_{\mathrm{mal}}^{(0)}$ of interface rigidity is transformed into the degree of freedom of point $Q$ on the relative interface through coordinate transformation:

$$
\begin{aligned}
u_{\mathrm{mal}}^{(0)} & =Y_{\mathrm{Q}} u_{\mathrm{mal}}^{(0)}=Y_{\mathrm{Q}} R_{1}^{+} u_{c}, \\
Y_{\mathrm{Q}} & =\left[\begin{array}{cc}
E & -L_{\mathrm{Q}} \\
0 & E
\end{array}\right],
\end{aligned}
$$

where $L_{Q}$ is the coordinate of point $Q$ and $R_{1}^{+}$is the Moore-Penrose inverse matrix of $R_{1}$. When the number of molecular interfaces is more, the subinterface can be uniformly defined as rigid interface. All subregions in the interface can be regarded as rigid equivalent. The $i$-th subinterface can be written as rigid equivalent:

$$
u_{\mathrm{mal}}^{(\mathrm{Q})}=Y_{\mathrm{Q}} R_{i}^{+} u_{c i}=Y_{\mathrm{Q}} R_{i}^{+} B_{i} u_{c},
$$

where $B$ is the Boolean matrix, the gas element is 0 or 1 , $R_{i}$ is the submatrix of $R_{1}$, and the submatrix satisfies $R_{i} \subset R_{1}$ and $U_{c i} \subset U_{c}$. Finally, combined with $U_{c}$ and $u_{\mathrm{mal}}^{(Q)}$ of the freedom degree of the interface rigid equivalent freedom degree and $u_{\mathrm{mal}}^{(\mathrm{Q})}$ of the equivalent freedom degree of all interfaces, the flexible equivalent freedom degree of the overall interface is formed:

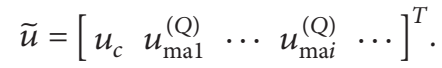

3.5. Application of Frequency Response Function considering Rotation Degree of Freedom. In the frequency domain substructure synthesis method to obtain the frequency response function in the angle of degrees of freedom of information, related scholars have carried out extensive research, Duarte and Ewins [40] using finite difference method, the measured component is obtained by matrix transformation theory of degree and frequency response function, and the degree of test methods are summarized. Varoto et al. [41] summarized the methods of obtaining the frequency response function of the degree of freedom of rotation and summarized some methods of obtaining the degree of freedom of rotation. Under the assumption that the area near the measured frequency range is rigid, Bregant and Casagrande [42] deduced the frequency response function considering the degree of freedom of rotation under the action of unit bending moment by using the frequency response function of unit excitation and matrix expansion technique.

Helderweirt et al. [43] used the translational acceleration measured by additional mass and vibration sensors to obtain the rotational degree of freedom of the measuring points and applied it to the measurement of the rotational degree of freedom of suspension components. Avitabile and O'Callahan [44] obtained FRF data including rotational degree of freedom by extending the residual modes and identifying the modal parameters. Silva et al. [45] through test research, such as in a complete frequency response function matrix, obtained $75 \%$ is the degree of frequency response function data, this paper discusses the thinking rotational degree of freedom in the frequency response function, and the importance of the substructure synthesis method is pointed out that ignoring degree will be one-sided frequency response function matrix, even the wrong frequency response function results. Considering the difficulty of direct measurement system and torque excitation, Silva and Montalvão et al. $[45,46]$ acquired the rotational degree of freedom by adding the T-block method. The operation method of the T-block aided test is to design a rigid T-block for measurement and make the rigid T-block rigidly connect with substructure and then apply excitation and pick-up response on the additional T-block. The frequency response function decoupling method is used to obtain the information of the frequency response function of the degree of freedom of rotation. There are two methods for measuring the degree of freedom of rotation of acceleration, one is the finite difference method, and the other is the T-block method. In [47], two methods and principles for measuring the degree of freedom of rotation are given, and the frequency response function matrix of the finite difference method is derived. The sketch of measuring the degree of freedom of rotation is shown in Figures 5-7. Model condensation and expansion technology has also been applied by more and more scholars to compensate the degree of freedom of frequency response function. Avitabile and O'Callahan [44] took into account the influence of residual number, used the method of system equivalent condensation and expansion to estimate the unmeasured frequency response function directly, and expanded the frequency response function matrix. The design substitutes such as van der Seijs et al. [48] are integrated with unknown substructures, and the rotational degree of freedom information of unknown substructures is obtained by the structural decoupling method. KLERK et al. [49] 


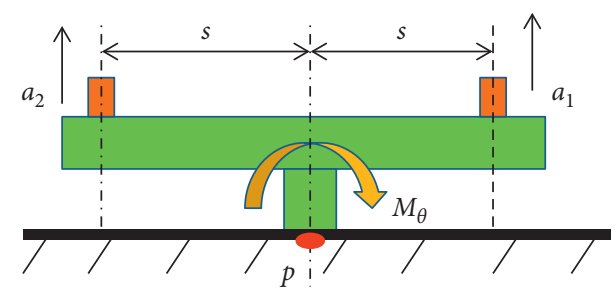

FIGURE 5: Finite difference method for measuring rotational degree of freedom.

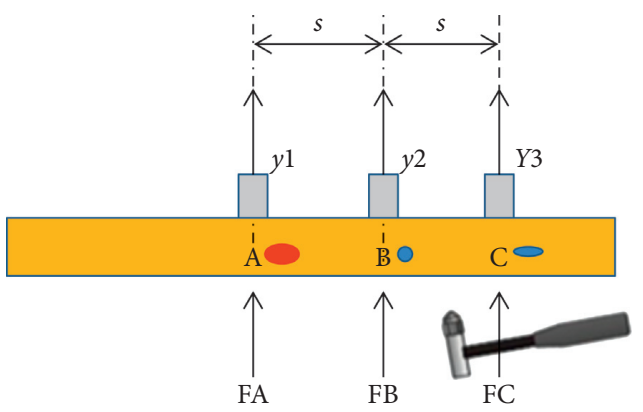

FIgURE 6: T-block method for angular acceleration measurement.

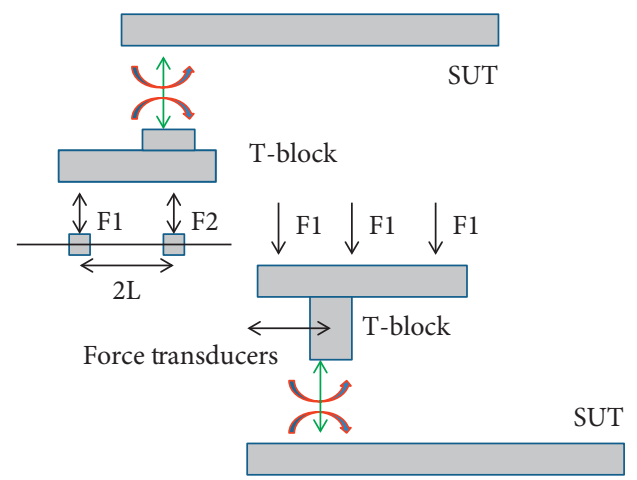

FIGURE 7: Method of applying bending moment.

proposed an equivalent multipoint connection method (EMPC), which assumes that the interface of the connection is rigid. According to the rigid body motion theory, the rigid point of the lifting part is equivalent; that is, the degree of freedom of the interface of the connection is converted to a rigid point with virtual six degrees of freedom. The rotation degree of freedom of the interface is included, and the original frequency response function is filtered by using the transformation relation. Under the condition of approximate rigidity of the whole interface, this method can improve the comprehensive accuracy of the response function.

Although some progress has been made in the research on the rotational degree of freedom information of FRF, there is no unified solution to the problem of precision reduction caused by the absence of rotational degree of freedom FRF. Equivalent multipoint method proposed by Klerk and Rixen provides a new way to extend the frequency response function of rotational degree of freedom. At the same time, the flexible equivalence of the interface is used to divide the interface into regions and form different interfaces. The rigid equivalence method of each subinterface is used to describe the flexible characteristics of the interface, so as to improve the accuracy of the rotational degree of freedom frequency response function of the missing interface.

\section{Model Modification of Substructure}

4.1. Model Modification Based on Neural Network. For complex substructures, matrix and parametric model modification cannot fundamentally overcome difficulties caused by measurement errors. In recent years, artificial neural network algorithms are increasingly favored by researchers. The basic process of neural network model correction technology is as follows: firstly, determine the structure type of neural network and select the parameters of neural network and then standardize the input conditions of learning samples, determine the initial weights, and input the number of training samples, so the neural network model is obtained. Finally, input the structural parameters obtained from the experiment into the model and the model parameters are corrected by controlling the error range. The types of neural networks commonly used in model modification of substructure mainly include feedforward neural network, multilayer perceptron neural network (MLP) (Figures 8 and 9), BP neural network, and radial basis function neural network (RBF). Pandey and Barari [50] modified the model by using multilayer perceptron neural network and error reversal algorithm. Atalla and Inman [51] and Levin et al. [52] used radial basis function neural network to modify the models of one-dimensional frame structure and two-dimensional plate structure, respectively, through the measured structure frequency response function.

Model modification and identification based on the iterative neural network, dual propagation neural network, probabilistic neural network, and fuzzy neural network for the complex structure has made some progress in recent years, but due to the complex topology of these neural networks, few of them can be applied [53-55]. In the process of using neural network to modify the model, the modified input parameters and output parameters have a great impact on ANN's network generalization ability. The type and number of input and output parameters will affect the number of neurons in the neural network, thus affecting the complexity of the neural network model. Through the measured Fourier spectrum response of the structure, $\mathrm{Wu}$ et al. [56] equated it into 200 spectral points, each of which corresponds to the neurons of the BP neural network. There was a lot of redundant information of input parameters, which led to the unsatisfactory effect of model recognition. Atalla and Inman [51] and $\mathrm{Lu}$ and $\mathrm{Tu}$ [57] analyzed the shortcomings of the above method and classified the spectral points of the frequency response function. They believed that the data value near the resonance frequency of the frequency response function was relatively large, and the credibility of the rest frequency range was reduced under the influence of the test noise. The frequency band with high credibility was integrated separately, and the integral value of each 


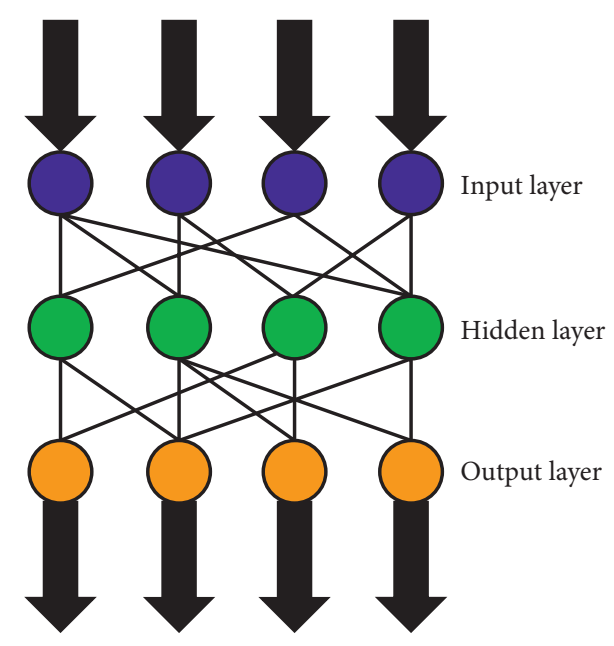

FIGURE 8: A typical feedforward neural network.

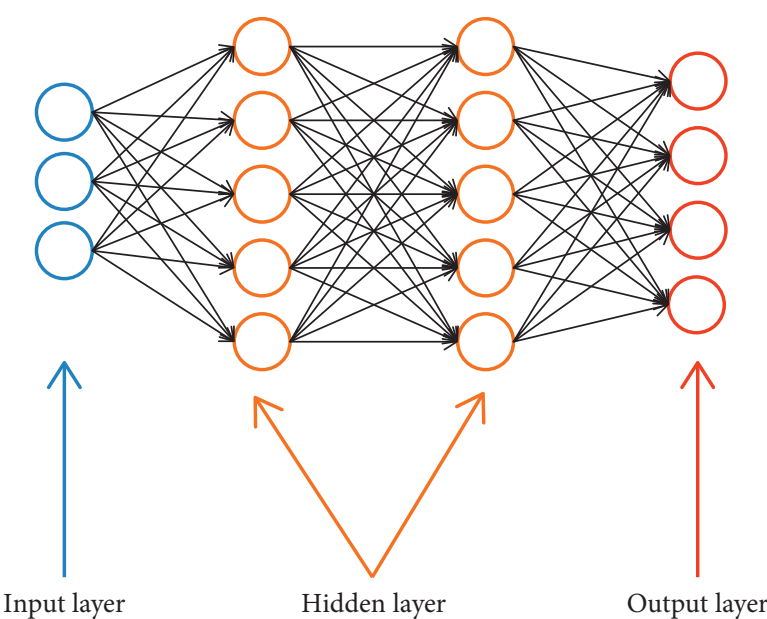

FIgURE 9: Multilayer perceptual neural network.

frequency band was taken as the model modification parameter. This method greatly reduces the number of neurons in the input layer and retains the important information of the original data. Tsou and Shen [58] applied the change in dynamic residual vector as the input of the $\mathrm{BP}$ neural network to modify the model and verified the effectiveness of the method with a spring-mass system with multiple degrees of freedom. Yun and Bahng [59] decomposed the whole structure into substructures, converted various factors that affect the error into the submatrix magnification factor of the substructure, and modified the model by adjusting the magnification factor. The results show that the method has a certain antinoise capability. Xu et al. [60] used the method of partial modification to modify the model of multilayer shear structure. This method first identifies the order and topological form of the structural model, then takes the first modified parameter into the second parameter as the input parameter of BP neural network, and gradually generates training and test samples to train the second BP neural network. The advantage of this method is that it does not need to solve the structural eigenvalue problem when generating training samples. Chang et al. [54] introduced the orthogonal matrix method in the selection of input parameters of neural network. This method can significantly reduce the sample set of network training and improve the accuracy and efficiency of calculation. Although the neural network-based model modification method has some advantages compared with the traditional model correction technology, some technical problems have not been solved. For example, the selection criteria of neural network model, parameter determination of hidden layer neurons, and initial weight of neurons lack sufficient theoretical guidance, especially for the complex models. Because the types of input parameters and the range of variation in the parameters in the complex model are very different, it is necessary to carry out complex preprocessing and parameter normalization for various types of input parameters, as well as sample set construction and selection. Secondly, with the increase in the modified parameters of the complex model, the corresponding training sample combination and training set show geometric progression growth, which is also a great test to the calculation accuracy and correction efficiency.

4.2. Model Modification Based on Modal Sensitivity. The basic idea of parametric modification method for dynamic substructure is to construct the error objective function between the theoretical model and the actual model, and then select the correction quantity or correction matrix to minimize the error of the correction quantity, so as to realize the parametric modification of dynamic substructure. The variables commonly used for parameter correction mainly include mass, stiffness, and damping matrix. By using the orthogonal criterion and solving the characteristic equation of the model, Fox and Kapoor [61] derived the first-order sensitivity calculation formula for the eigenvalue and eigenvector of the linear structure and the design parameters. After that, Rogers [62] and Garg [63] improved Fox's research results and promoted the further development of modal sensitivity correction methods. Nelson [64], Lim, and Junkins [65] simplified the sensitivity formula of feature vector from the angle of difference algorithms and numerical calculation and simplified the calculation amount of model correction. By constructing the square of the model correction in the objective function and the weighted sum of the residual vectors of the eigenvalue sensitivity, Zhang et al. [66] applied the Newton iteration method to correct the eigenvector after each iteration and applied the modified eigenvector to the next iteration. Farhat and Hemez [67] proposed a method to modify the model one by one in the substructure of the element. By constructing the modal dynamic residual of the element, the residual was minimized to the objective function, and the iterative solution was carried out to obtain the extended mode, which was then substituted into the next iteration to differentiate each parameter and obtain the modified parameters.

Since the modification method of modal sensitivity analysis needs to measure the natural frequency and mode shape of substructures, when the adjacent modes of the model are relatively dense and it is difficult to separate the modes, this method often encounters difficulties in 
identification. In order to overcome this difficulty, a method called frequency response function correction (RFM) without modal testing and identification is proposed.

4.3. Model Modification Based on RFM. The basic methods of frequency response function can be summarized as follows: the residual correction method based on frequency response function, frequency response method based on modifying design parameters, and correction method based on sensitivity $[68,69]$. The main research results are as follows: Natke [70] first applied the weighted least square technique to the model modification technique of frequency response function. After that, Foster and Mottershead [71], Link [72], and Friswell and Penny [73] refined the method. By using Taylor series to expand the dynamic stiffness matrix, Larsson and Sas [74] proposed a new deformation of the equation residual, which corrected the parameter error problem and obtained a more accurate frequency response function matrix. Lin and Ewins [75] obtained the transfer matrix FRF function by experimental means and obtained the transfer function FRF obtained by the finite element mathematical model by two means for error analysis and minimized the error to modify the model's mass matrix and element stiffness. Visser and Imregun [76, 77] further studied the modified element matrix and proposed two methods to modify the frequency response function matrix, one is the linear perturbation of the element mass, element stiffness, and damping matrix based on the model, and the other is the modification based on the residual errors of the input and output. Link and Zhang [78] constructed residual information in the form of structural force response to modify the model. Zang et al. [79] defined the correlation coefficients of shape and amplitude, respectively, to evaluate the similarity between the target frequency response function and the prediction frequency response function and modified the model of sensitivity of design parameters according to the correlation coefficients. Through the linear correlation between the row and column of the sensitivity matrix, Kwon and Lin [80] introduced the parameter independent index (PDI) like the MAC matrix to guide the selection of the optimal frequency point data for model modification and carried out the model modification of the selected frequency point. Imregun et al. [81] further studied the method, systematically derived the relationship between the frequency response function and the dynamic stiffness matrix of the model, introduced the error parameters of design parameters, measured dynamic stiffness and theoretical dynamic stiffness into the equation, and solved the correction through the modified parametric equation. Lin and Zhu [82] proposed a method to modify the finite element model by using the frequency response function of unit excitation and verified the validity and adaptability of this method. Adhikari and Friswell [83] used the method of frequency response function to identify and modify the damage of the structural model and verified the validity of the method. Aiming at the error between the measured frequency response function and the measured frequency response function calculated by the finite element model,
Larsson [84] improved the model modification method of the frequency response function, studied the problems caused by the large error or the measured frequency response function noise, and effectively reduced the error between the measured frequency response function and the finite element calculation. Steenackers and Guillaume [85] studied the parameter selection and control method of model modification, improved the existing modification method, took the measured frequency response function error and noise and other uncertainties into the correction process, and improved the correction accuracy by this method.

The advantage of the RFM method is that it does not need modal analysis and avoids the error caused by highorder modal dense truncation. The disadvantage of RFM is that it needs a complete value of frequency response function. Before modification, the measured frequency response function needs to be expanded or the model needs to be condensed. In addition, the measured frequency response function must be applied with controllable excitation, which is difficult to realize for the complex and large structure model. By comparing the characteristics of the modified eigensensitivity method of the model with that of RFM, Modak et al. [86] found that the modified eigensensitivity method had more stringent requirements on the number of measurement points due to the influence of computational convergence. When the measurement points are relatively small, the characteristic sensitivity algorithm needs to conduct error calculation to narrow the selection range of modification parameters. When the number of modes is large, the accuracy of sensitivity modification is higher than that of the frequency response function correction algorithm. When the number of modes involved in the modification decreases gradually, the convergence of the solution set can be guaranteed by the frequency response function algorithm, but the convergence of the solution set cannot be guaranteed by the sensitivity modification algorithm.

4.4. Model Modification Based on GA and SA. Since the parameter modification method of dynamic substructure can be equivalent to solving the optimization problem of objective function, the optimization algorithm is very important in the calculation of model modification. The objective function involved in solving the model modification problem of complex structure usually has the characteristics of high nonlinear and multiple local extreme points. Therefore, traditional optimization algorithms, such as Newton iterative method, gradient method and difference method, are difficult to find local optimal solution, while genetic algorithm (GA) and simulated annealing method (SA) have great advantages in solving model correction of local optimal solution. The fitness function of the genetic algorithm, also known as the evaluation function, is used to judge the degree of fitness of individuals in the group. It is based on the objective function of the problem to be evaluated. In the process of searching evolution, the genetic algorithm generally does not need other external information but only uses evaluation function to evaluate the merits 
of individuals or solutions and serves as the basis for future genetic operations. Genetic operation is the practice of simulating genetic inheritance in organisms. In the genetic algorithm, after the initial population is formed through coding, the task of genetic operation is to impose certain operation on the individuals of the population according to their environmental fitness, so as to realize the evolution process of survival of the fittest. Genetic operations include the following three basic genetic operators: selection, crossover, and variation. Simulated annealing is a stochastic optimization algorithm based on the iterative solution strategy of Monte Carlo. It is a serial structure optimization algorithm, which can avoid falling into local minima and then tend to global optimum by giving the search process a probabilistic jump with time variation and finally tend to zero. Using the GA algorithm, Teughels et al. [87] proposed a coupled feature local optimization method and applied it to finite element model modification, which solved the problem of local feature optimization of complex dynamic structures that could not be solved by traditional methods. Mares and Surace [88] constructed the force residual error equation by measuring the inherent characteristics of the structure, optimized the residual error parameters through the GA optimization algorithm, adjusted the structural parameters, and obtained a good result. Their work also shows that the GA algorithm has a certain antinoise ability to the measured signal. Friswell et al. [89] used the comprehensive sensitivity optimization algorithm and GA algorithm for damage identification of the structure, according to the GA algorithm to find the optimal solution set of the location of structural damage, and then use the characteristic sensitivity algorithm for structural damage degree of structural sensitivity evaluation. Dunn [90] compared the difference between the objective function method of frequency response function and GA algorithm on global optimal and local optimal solutions and showed that the GA algorithm was more suitable to obtain the global optimal solution set through the spring-mass structure of two degrees of freedom. Zimmerman et al. [91] proposed an effective genetic mutation operator and genetic algorithm fitness function to make genetic search more reasonable. They analyzed the influence of genetic mutation operator on model modification and concluded that the changing genetic mutation operator makes the genetic population have more diversity, and the model modification is more accurate. Levin and Lieven [92] modified and optimized the dynamic model with GA algorithm and SA algorithm, respectively, compared the computational iteration speed and optimal solution set of the two genetic algorithms, and concluded that the SA algorithm was better than the traditional SA algorithm in performance. Chou and Ghaboussi [93], Rao et al. [94], and Au et al. [95] studied the application effect of the GA algorithm in model modification and structural damage assessment and evaluated the application prospect of the GA algorithm.

4.5. Matrix Model Modification. Matrix model modification is to directly modify stiffness matrix and mass matrix based on orthogonality condition by using finite element analysis results and test data, so as to achieve the consistency of calculation results and test results. One of the earliest methods of matrix model modification is the reference basis method. This method assumes that the mass matrix, stiffness matrix, and modal matrix have two terms as variables and one term is fixed. The modification method is to construct the objective function of the model by using the least square principle and to optimize and modify the parameter matrix through the Lagrange multiplier method and constraint conditions.

4.6. Objective Function Modification Method. By testing the natural frequency and the corresponding incomplete modal information, Berman and Nagy [96] used the characteristic equation of the system and the stiffness and mass matrix of the model to expand the modal and constructed the following objective function:

$$
J_{m}=\left\|M_{A}^{-1 / 2}\left(M-M_{A}\right) M_{A}^{-1 / 2}\right\|+\sum_{i=1}^{m} \sum_{j=1}^{m} \lambda_{i j}\left(\phi^{T} M \phi-I\right)_{i j},
$$

where $M_{A}$ is the mass matrix before modification, $\lambda_{i j}$ is the Lagrange factor, and $m$ is the measured modal density. The modified mass matrix can be obtained by optimizing the objective function as follows:

$$
M=M_{A}+M_{A} \phi m_{A}^{-1}\left(I-m_{A}\right) m_{A}^{-1} \phi^{T} M_{A} .
$$

Among them, $m_{A}=\phi^{T} M_{A} \phi$. The modified mass matrix satisfies the orthogonal condition, and the stiffness matrix and mass matrix satisfy the symmetry condition. Based on the modified matrix, the following objective function is reconstructed:

$$
\begin{aligned}
J_{m f}= & \left\|M_{A}^{-1 / 2}\left(K-K_{A}\right) M_{A}^{-1 / 2}\right\|+\sum_{i=1}^{p} \sum_{j=1}^{m} \lambda_{k i j}(K \phi-M \phi \phi)_{i j} \\
& +\sum_{i=1}^{m} \sum_{j=1}^{m} \lambda_{o i j}\left(\phi^{T} K \phi-\Lambda\right)_{i j}+\sum_{i=1}^{p} \sum_{j=1}^{i} \lambda_{s i j}\left(K-K^{T}\right)_{i j}
\end{aligned}
$$

where $P$ is the degree of freedom of the model. The modified stiffness matrix is obtained by optimizing the reconstructed objective function $J_{m f}$ :

$$
\begin{aligned}
& K=K_{A}+\left(\Delta+\Delta^{T}\right), \\
& \Delta=\frac{1}{2} M \phi\left(\phi^{T} K_{A} \phi+\Lambda\right) \phi^{T} M-K \phi^{T} \phi M .
\end{aligned}
$$

The advantage of the Berman method is that it does not need to iterate and repeat the analysis of eigenvalues but only needs to invert the generalized mass matrix, which is suitable for complex structures with a higher degree of freedom. The disadvantage is that the mass matrix and stiffness matrix obtained by this method change the sparsity performance of the original matrix, and the matrix's main diagonal elements will have imaginary elements and negative stiffness or mass. 
4.7. Multiple Modified Reference Basis Method. The traditional reference basis method focuses on the physical authenticity of the model, but the reference standard is not comprehensive enough and has some shortcomings. Due to the increase in the modified parameters, most of the parametric modification methods need to adopt the indirect method in the calculation, and the calculation efficiency is low. Halevi and Bucher [97] combined the two methods and proposed a generalized weighted reference basis method of multiple modification. This method considers the influence of the connection information loss function on the stiffness matrix of the modified model, which can not only satisfy the test structure but also keep the connection information of the original model, that is, the loss function, to a minimum. The process of correction is divided into two steps: the first step is to obtain an unconstrained modified stiffness matrix $K$ according to the reference method:

$$
\begin{aligned}
K= & K_{A}-\left(K_{A} \phi-M \phi \phi\right) R^{T}-R\left(K_{A} \phi-M \phi \phi\right)^{T} \\
& +R\left(\phi^{T} K_{A} \phi-\Lambda\right) R^{T} .
\end{aligned}
$$

Among them, $R=W \phi\left(\phi^{T} W \phi\right)^{-1}, \Lambda$ is the eigenvalue matrix, $W$ is the coefficient matrix, and it is selected according to the residual eigenvalue of the model. The $K$ value obtained in the first step makes the modified model highly consistent with the experimental value, but the connection information of each connection unit in the model is missing. The second step is to look for a new modified matrix $K_{n}$, so that the new modified matrix satisfies the original contact information of the model and also satisfies the Frobenius module meaning approaching the value of $K$. Based on the original model stiffness matrix $K_{A}$, the perturbation of physical parameter $\alpha$ is linearly expanded. The principle of perturbation satisfies the following relations:

$$
\min _{\alpha}(J)=\left\|K_{n}-K\right\|_{F}^{2}=\left\|\sum_{i=1}^{p} \alpha_{i} \frac{\partial K_{A}}{\partial \alpha_{i}}-\left(K-K_{A}\right)\right\|_{F}^{2} .
$$

The value of the weighting coefficient is adjusted continuously to make the modified stiffness value $K_{n}$ meet the minimum connection information loss function, and the modified function is obtained:

$$
\min \left(J_{n}\right)=\frac{\left\|K-K_{n}\right\|_{F}}{\left\|K_{n}\right\|_{F}}
$$

The defect of matrix modification method is that it cannot preserve the connection information of the original model. The improved algorithm is cumbersome in the selection of modification parameters and weight coefficients, which affects the calculation efficiency of the model. In addition, the incomplete measurement data lead to the nonuniqueness of the modification result. Matrix modified extension method requires the mass matrix and stiffness matrix of the original model, while they themselves are generally fuzzy parameters that need to be modified so that the extension and the modification are coupled. Therefore, the development of the matrix correction method is still far behind other optimization algorithms.
4.8. Problems in Model Modification. Model modification technology is the basis of dynamic identification and parameter evaluation of structure. Great progress has been made in recent years, but there are still the following problems in the field of model modification:

(1) At present, most of the modification methods are basically aimed at a specific problem, which has a narrow adaptation range and is difficult to be generalized. Large complex structures have higher degrees of freedom, more correction parameters, and there are a large number of assumptions of specific boundary conditions in the model, making the modification difficult. The accuracy and efficiency of the modified algorithm need to be solved.

(2) As a kind of inverse dynamic problem, model modification results often depend on the processing of measured data. Modal truncation and measurement error have great influence on the correction results. How to improve the measurement accuracy and reduce the measurement noise error are also important factors affecting the model modification accuracy.

(3) For matrix modification, the defect of the algorithm is that it cannot preserve the connection information of the original model. Although the improved algorithm can guarantee the connection information, the selection of correction parameters and weighting coefficients is complicated and cumbersome, which affects the calculation efficiency. In addition, there are problems of matrix iteration, convergence, illconditioned matrix, and the selection of modified objective function. If there are too many parameters to be modified, the calculation of matrix is huge, which hinders the application of the physical parametric method.

(4) Parametric modification method can guarantee the connection information of the original model remains unchanged, but there is a problem that the modified parameters are larger than the number of independent equations, which lead to the problem that the solution of the equation is not unique or nonexistent. Therefore, it is difficult to find an efficient error location algorithm to narrow the range of parameter selection.

(5) The main problem of the modification method of the neural network model is that the selection of the neural network topological structure is currently lack of relevant theoretical guidance. For large complex structure models, when the modification parameters increase, the network structure becomes complicated and the training cost and training time increase geometrically; the computational efficiency cannot be guaranteed. Therefore, there are still some problems in the application of this method to model modification of large and complex structures. 


\section{Test and Engineering Application}

5.1. Test. The test verification of substructure synthesis and modification method usually has two methods: one is to carry out experimental verification on actual physical objects and measure the dynamic parameters of the structure, such as mode, transfer function, and dynamic response, to obtain the dynamic performance of the structure. The other is to use the numerical simulation method to establish an equivalent model for the physical object to simulate the dynamic response characteristics of the structure. The purpose of test verification is to test the solution accuracy and efficiency of theory and method. In terms of comprehensive validation of substructures, Donders et al. [28] used the assembly model of composite board to verify the reduction method of the degree of freedom of the interface of substructure connection. The characteristic frequency error between the reduced assembly model and the initial model was $<0.1 \%$ in the low and medium frequency range, and the MAC matrix diagonal value was $>0.98$, which verified that the numerical simulation had high accuracy for the simple structure. In literature [98], Jetmundsen et al. [32] used frequency-domain synthesis method to conduct experimental research on the space steel frame structure, and the difference in frequency-domain synthesis between indirectly obtained rotational degree of freedom information and translational degree of freedom information was compared. Drozg et al. [99] proposed a solution method based on frequency response function matrix containing angle freedom degree and extended the response model with modal model. Through the dynamic response test of steel beam (as shown in Figure 10 [99]), it was verified that the solution precision of the angle freedom degree synthesis method was higher than that of the translational freedom degree synthesis method. In the document [100], in view of the existing frequency response function synthesis method which only considers the lack of vibration isolator stiffness and damping characteristics, the FBS substructure synthesis algorithm (frequency response function-based substructuring) is improved, introducing quality FBS algorithm and algorithm applicability is verified by floating raft isolation system test, and improved FBS algorithm has higher accuracy in lowfrequency range with the actually measured value. In terms of substructure model modification, Guvenc et al. [101] applied the response function method (RFM) to the nonlinear system, solved the system characteristic sensitivity through the finite element method, and realized the modification of the nonlinear model structure. With the help of the nonlinear T-beam structure test (as shown in Figure 11 [101]), the error rate between the modified model and the measured results is verified to be $<3 \%$, which meets the accuracy requirements of engineering applications. Wang et al. [102] used the composite sandwich beam test (as shown in Figure 12) and model modification agent model, through the FEA model and agent model to establish a mapping relationship, with the help of the KRIGING model instead of the finite element model of iterative calculation to seek optimal solution parameters. Moreover, it was verified that the KRIGING model has a high modification accuracy for low frequency $0-200 \mathrm{~Hz}$ and a large error when the frequency is greater than $400 \mathrm{~Hz}$. In the application of model modification optimization algorithm (genetic algorithm and neural network algorithm), Wang and Niu [103] used the genetic algorithm to modify the finite element model by taking mode frequency and mode shape as objective functions. Through the modal test of beam with variable cross section, it was verified that the mode error rate of the GA algorithm was $\leq 0.22 \%$ within the low frequency $100 \mathrm{~Hz}$. However, with the increase in optimization parameters, the convergence speed and modification accuracy are reduced.

The test verification of substructure synthesis and modification is mostly based on simple structures such as composite plates and beams. Since the simple structure does not need complex boundary processing and the structure modes are uniformly distributed in the relatively low-frequency range without a large number of dense mode distributions, which is convenient for system identification, the majority of test verification results have a small error in the low-frequency range of $0-200 \mathrm{~Hz}$. For the system errors in the low-frequency range, the design parameters in the original model can be modified by means of modal synthesis, frequency response function (FRF) synthesis, transfer matrix, and GA algorithm, and the system errors can be reduced by optimizing design variables. At present, it is still difficult to verify the subsystem method of a large complex structure. The main influencing factors are equivalent conversion between theoretical model and experimental model, treatment of complex boundary conditions of theoretical model, and truncation error of higher-order modes. Therefore, the development and verification of complex structure subsystem synthesis algorithm is one of the following research emphases in this field.

5.2. Engineering Application. The substructure synthesis and modification method can quickly realize the dynamic design and modification of the structure, effectively reduce the dynamic analysis and calculation scale, has a broad application prospect in engineering, and has been widely used in automobile, aviation, ship, and other transportation fields in recent years. Gagliano [104] applied the transfer matrix synthesis method to decompose the whole vehicle into substructures such as steering system, body system, and suspension system, built a single steering system test bed (as shown in Figure 13 [104]) and a preloaded tire test bed, and predicted and modified the dynamic response of the whole vehicle through the substructure frequency response function. The test results show that the prediction accuracy within $200 \mathrm{~Hz}$ can meet the actual needs of engineering, and the prediction accuracy of medium and high frequency is relatively low. The main factors that affect the error are the stiffness of rubber bushing, the nonlinearity of damping, and the measurement noise. John et al. [105] applied the substructure modification method for helicopter model verification, and the whole structure of the helicopter is decomposed into subsystems such as passenger cabin, tail arm, and tail wing. In the modification test, 29 response points and 4 excitation points are adopted to test the transfer 


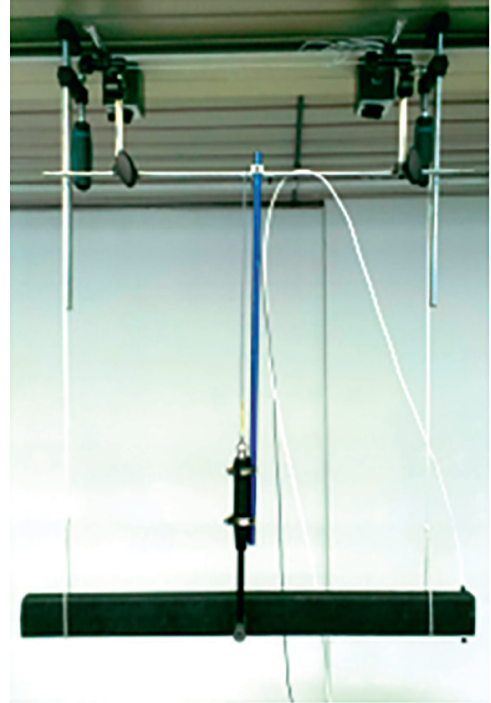

(a)

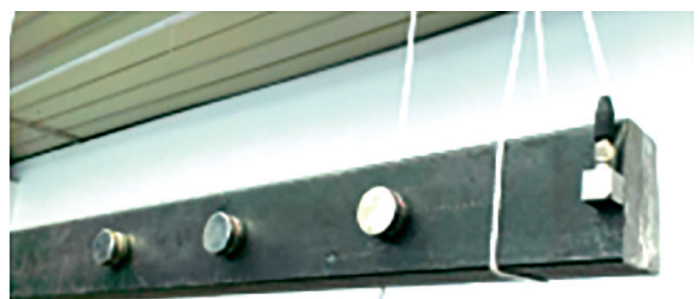

(b)

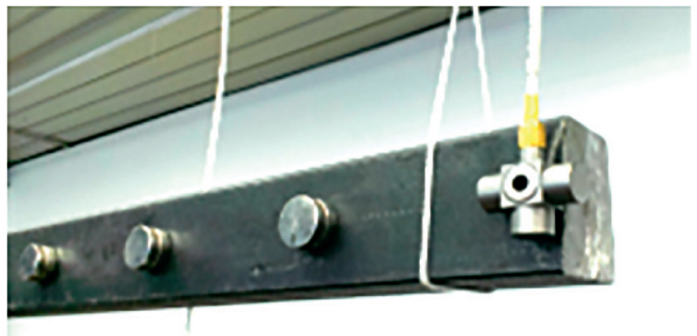

(c)

Figure 10: Dynamic response test device of steel beam [99].

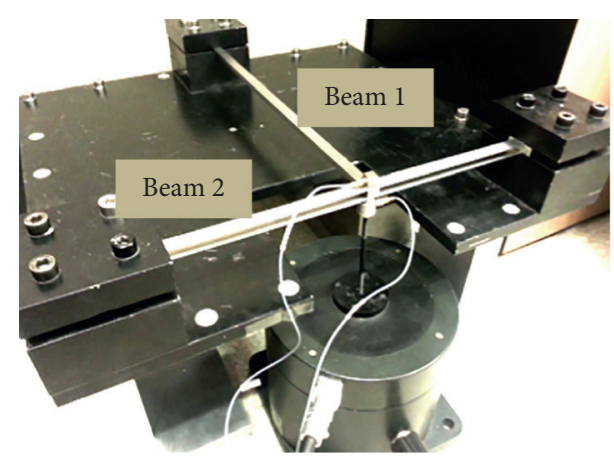

FIGURE 11: Experimental device for nonlinear beam structure [101].

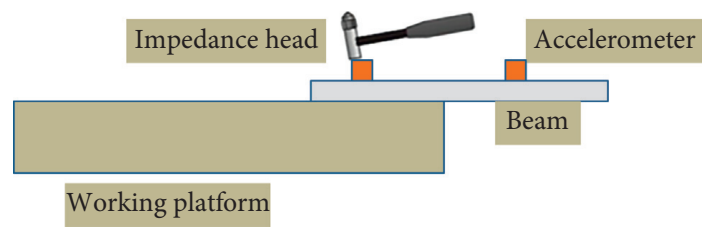

FIGURE 12: KRIGING model modification test device.

function of cross-point excitation. By modifying the transfer function between subsystems, the overall model is modified. Kim et al. [106] combined the comprehensive method of frequency response function and transfer path analysis (TPA) technology to predict and optimize the noise transfer function (NTF) in the car. The test process is shown in Figure 14. In the optimization process, the sub-structure frequency response function and TPA program are adopted to change the local stiffness of the car body and reduce the acoustic radiation efficiency so as to control the in-car noise. Sakai [107] used the FBS substructure frequency response synthesis method to predict the in-car response caused by engine noise. Although the substructure synthesis and modification method has been widely applied, the prediction accuracy of complex structures including nonlinear stiffness and damping is lower, and the prediction accuracy of medium and high frequency models is lower. The main reason is that the modal density of medium and high frequency structures' substructure modal identification has certain errors for dense modes, and there is a strong coupling relationship between medium and high frequency structures and acoustics. Coupling loss factor, damping loss factor of structure and acoustics, modal density, and other parameters directly or indirectly affect the prediction accuracy of the model. Combining the finite element model modification method with medium and high frequency statistical energy correction method is one of the ideas to solve medium and high frequency problems. The calculation accuracy depends on the subsystem partition of the hybrid model and the 


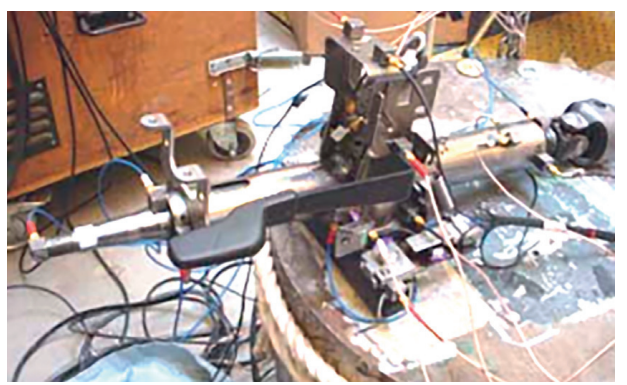

Figure 13: Substructure test bed of the steering system [104].

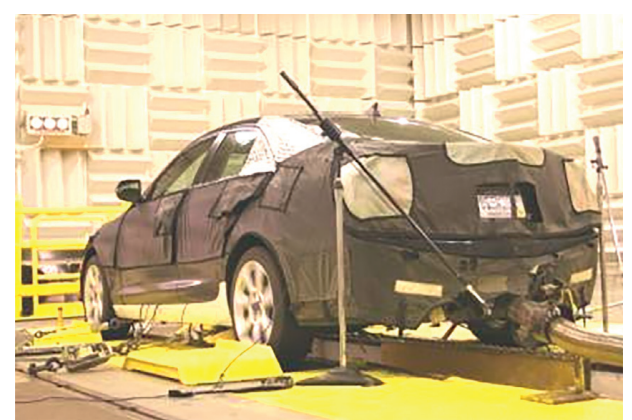

FIgURE 14: Application case of vehicle noise prediction.

determination of the energy transfer relation between the statistical energy boundary and the finite element boundary.

\section{Conclusion}

To sum up, the methods of vibration substructure synthesis and modification have made great progress in theoretical research and application in recent years, with new methods and algorithms emerging one after another. However, the comparative analysis of the above aspects shows that the following problems still need to be solved in the field of substructure synthesis and model modification. In addition, this paper also gives the reader some advice for further research.

In terms of substructure synthesis,

(1) Modal synthesis, condensation polymerization, and mechanical impedance admittance have some limitations in application and are not suitable for very large complex structures and structures with complex boundary conditions. The error caused by highorder mode truncation exists in mode condensation polymerization.

(2) In the frequency-domain substructure, the method proposed by Jetmundsen and Ren only considers rigid connections and is only applicable to independent substructures. The method of solving the frequency response function matrix is only limited to two substructures and is not applicable to the synthesis of frequency response functions with more than three substructures. In the process of solving the mechanical impedance and admittance method, each substructure frequency response matrix has ill-posed matrix in the resonance region, which affects the fitting effect of the whole multisystem. It is a new trend in dynamic structure modeling to find a method for dynamic substructure, rigid coupling, flexible connection, considering mechanical impedance, incorporating residual compensation into substructure, and modifying substructure matrix.

(3) At present, there is no unified solution to the problem of accuracy reduction caused by the absence of angle freedom frequency response function. The equivalent multipoint method makes rigid equivalent connection and flexible equivalent connection to the interface to improve the accuracy of the angular freedom frequency response function of the missing interface, which will be the focus of further research.

In terms of substructure model modification,

(1) When it is difficult to define the large complex structure or the boundary conditions of the structure, the accuracy of the modified algorithm is limited.

(2) The matrix model modification and the parametric modification method have some defects in preserving the join information of the model, and the independent solution set is not unique.

(3) It is an urgent task to develop a new universal multidegree-of-freedom structure modification algorithm for large and complex structures. On this basis, the research focus of model correction should focus on how to take into account the physical meaning and connection information of the original model as well as the accuracy and efficiency of the correction algorithm and make sure the error tolerance is strong.

Some advice for further research is listed as follows:

(1) At first, construct a substructure synthesis method that can be used to set up the connection between measurement mode shape parameters and the substructures.

(2) To account for the effect of connect dynamic characteristics on structural synthesis, introducing the correlation between substructures and the dynamic characteristics of the connecting parts and considering the substructural synthesis method with connection dynamic characteristics.

(3) A reasonable mathematical model should be determined before the model is modified.

(4) Taking population obtained from the genetic algorithm as the initial values, the updating factors can be obtained by substructure method. This method can solve the problem that the solution tends to trap in local optimum when the number of updating factors is more and the measured modes are less.

\section{Conflicts of Interest}

The authors declare that they have no conflicts of interest. 


\section{Acknowledgments}

This work was partially supported by the National Natural Science Foundation of China (nos. 51275541 and 2017YFB0102603).

\section{References}

[1] A. Oktav, Ç. Yılmaz, and G. Anlaş, "Transfer path analysis: current practice, trade-offs and consideration of damping," Mechanical Systems and Signal Processing, vol. 85, no. 8, pp. 760-772, 2017.

[2] L. Keersmaekers, L. Mertens, R. Penne, P. Guillaume, and G. Steenackers, "Decoupling of mechanical systems based on in-situ frequency response functions: the link-preserving, decoupling method," Mechanical Systems and Signal Processing, vol. 58-59, no. 5, pp. 340-354, 2015.

[3] Y. Ren and C. F. Beard, "On substructure synthesis with FRF data," Journal of Sound and Vibration, vol. 185, no. 5, pp. 845-866, 2001.

[4] W. Liu and D. J. Ewins, "Substructure synthesis via elastic media," Journal of Sound and Vibration, vol. 257, no. 2, pp. 361-379, 2002.

[5] D.-H. Lee and W.-S. Hwang, "An identification method for joint structural parameters using an FRF-based substructuring method and an optimization technique," Journal of Mechanical Science and Technology, vol. 21, no. 12, pp. 2011-2022, 2007.

[6] D. D. Klerk, D. J. Rixen, and S. N. Voormeeren, "General framework for dynamic substructuring: history, review and classification of techniques," AIAA Journal, vol. 46, no. 5, pp. 1169-1181, 2008.

[7] S. N. Voormeeren, P. L. C. Van der Valk, and D. J. Rixen, "Generalized methodology for assembly and reduction of component models for dynamic substructuring," AIAA Journal, vol. 49, no. 5, pp. 1010-1020, 2011.

[8] R. R. Craig, "Coupling of substructures for dynamic analyses: an overview," in Proceedings of the 41st Structures, Structural Dynamics, and Materials Conference and Exhibit, pp. 15732000, Atlanta, GA, USA, April 2000.

[9] S. N. Hou, "Review of modal synthesis techniques and a new approach," Shock and Vibration Bulletin, vol. 40, no. 4, pp. 25-39, 1969.

[10] R. R. Craig and C.-J. Chang, "Free-interface methods of substructure coupling for dynamic analysis," AIAA Journal, vol. 14, no. 11, pp. 1633-1635, 1976.

[11] L. E. Suarez and M. P. Singh, "Improved fixed interface method for modal synthesis," AIAA Journal, vol. 30, no. 12, pp. 2952-2958, 1992.

[12] R. E. D. Bishop and D. C. Johnson, The Mechanics of Vibration, Cambridge University Press, Cambridge, UK, 1960.

[13] G. J. O'Hara, "Mechanical impedance and mobility concepts," The Journal of the Acoustical Society of America, vol. 41 , no. 5, pp. 1180-1184, 1967.

[14] J. H. Gordis, "Integral equation formulation for transient structural synthesis," AIAA Journal, vol. 33, no. 2, pp. 320324, 1995.

[15] D. J. Rixen and P. L. C. van der Valk, "An impulse based substructuring approach for impact analysis and load case simulations," Journal of Sound and Vibration, vol. 332, no. 26, pp. 7174-7190, 2013.

[16] W.-L. Dong, L. Liu, S.-L. Zhou, and S.-L. Chen, "Substructure synthesis in time domain with rigid-elastic hybrid joints," AIAA Journal, vol. 53, no. 2, pp. 504-510, 2015.
[17] R. J. Guyan, "Reduction of stiffness and mass matrices," AIAA Journal, vol. 3, no. 2, p. 380, 1965.

[18] J. C. O'Callahan, "A procedure for an improved reduced system (IRS) model," in Proceedings of the 7th International Modal Analysis Conference, Union College Press, Schenectady, NY, USA, January 1989.

[19] R. L. Kidder, "Reduction of structural frequency equations," AIAA Journal, vol. 11, no. 6, p. 892, 2012.

[20] J. O'Callahan, "System equivalent reduction expansion process (SEREP)," in Proceedings of the 7th International Modal Analysis Conference, Schenectady, NY, USA, January 1989.

[21] J. L. Raynaud, N. Bouhaddi, A. Perriot, and F. Van Herpe, "Updating complex structures by a robust multilevel condensation approach," Journal of Sound and Vibration, vol. 270, no. 1-2, pp. 403-416, 2004.

[22] D.-M. Tran, "Component mode synthesis methods using interface modes: application to structures with cyclic symmetry," Computer \& Structure, vol. 79, Article ID 209222, 2001.

[23] G. Battiato, C. M. Firrone, T. M. Berruti, and B. I. Epureanu, "Reduction and coupling of substructures via Gram-Schmidt interface modes," Computer Methods in Applied Mechanics and Engineering, vol. 336, pp. 187-212, 2018.

[24] B. Frank, "Multi-level substructuring combined with model order reduction methods," Linear Algebra and Its Applications, vol. 436, no. 10, pp. 3864-3882, 2012.

[25] S. H. Sung and D. J. Nefske, "Component mode synthesis of a vehicle structural-acoustic system model," AIAA Journal, vol. 24, no. 6, pp. 1021-1026, 1986.

[26] J. Lee, D. J. Thompson, H. H. Yoo, and J. M. Lee, "Vibration analysis of a vehicle body and suspension system using a substructure synthesis method," International Journal of Vehicle Design, vol. 24, no. 4, pp. 360-371, 2000.

[27] J.-B. Qiu, F. W. Williams, and R.-X. Qiu, "A new exact substructure method using mixed modes," Journal of Sound and Vibration, vol. 266, no. 4, pp. 737-757, 2003.

[28] S. Donders, B. Pluymers, P. Ragnarsson, R. Hadjit, and W. Desmet, "The wave-based substructuring approach for the efficient description of interface dynamics in substructuring," Journal of Sound and Vibration, vol. 329, no. 8, pp. 1062-1080, 2010.

[29] D. J. Ewins, "Modal test requirements for coupled structure analysis using experimentally derived component models," in Experimental Analvtical Modeling of Dynamic Structural Systems, pp. 31-47, ASME, New York, NY, USA, 1985.

[30] C. Q. Liu, "Combination of an improved FRF-based substructure synthesis and power flow method with application to vehicle axle noise analysis," Shock and Vibration, vol. 15, Article ID 830281, 10 pages, 2008.

[31] P. Holzwarth and P. Eberhard, "SVD-based improvements for component mode synthesis in elastic multibody systems," European Journal of Mechanics-A/Solids, vol. 49, pp. 408$418,2015$.

[32] B. Jetmundsen, R. L. Bielawa, and W. G. Flannelly, "Generalized frequency domain substructure synthesis," Journal of the American Helicopter Society, vol. 33, no. 1, pp. 55-64, 1988.

[33] Y. Ren and C. Beards, "A generalized receptance coupling technique," in Proceedings of the 11th International Modal Analysis Conference, pp. 868-871, Kissimmee, FL, USA, February 1993.

[34] M. Imregun and D. A. Robb, "Structural modification and coupling dynamic analysis using measured FRF data," in 
Proceeding of the 5th International Modal Analysis Conference, pp. 1136-1141, London, UK, April 1987.

[35] K. Y. Sanliturk and O. Cakar, "Noise elimination from measured frequency response functions," Mechanical Systems and Signal Processing, vol. 19, no. 3, pp. 615-631, 2005.

[36] Y. Ephraim and H. L. Van Trees, "A signal subspace approach for speech enhancement," IEEE Transactions on Speech and Audio Processing, vol. 3, no. 4, pp. 251-266, 1995.

[37] M. T. Johnson and R. J. Povinelli, "Generalized phase space projection for nonlinear noise reduction," Physica D: Nonlinear Phenomena, vol. 201, no. 3-4, pp. 306-317, 2005.

[38] Y. Hu and P. C. Loizou, "A subspace approach for enhancing Speech corrupted by colored noise," Signal Processing Letters IEEE, vol. 9, no. 7, pp. 204-206, 2002.

[39] K. Hermus, P. Wambacq, and H. V. Hamme, "A review of signal subspace speech enhancement and its application to noise robust speech recognition," Eurasip Journal on Advance in Signal Processing, vol. 2007, no. 1, pp. 1-15, 2006.

[40] M. L. M. Duarte and D. J. Ewins, "Rotational degrees of freedom for structural coupling analysis via finite-difference technique with residual compensation," Mechanical Systems and Signal Processing, vol. 14, no. 2, pp. 205-227, 2000.

[41] P. S. Varoto, M. Lofrano, T. R. Cicogna et al., "Moment mobility FRF measurement techniques," in Proceedings of the IMAC-XXIV Conference \& Exposition on Structural Dynamics, St. Louis, MO, USA, 2006.

[42] L. Bregant and D. Casagrande, "Rotational degrees of freedom data synthesis based on force excitation," in Proceedings of the International Seminar on Modal Analysis, ISMA25, Leuven, Belgium, 2001.

[43] S. Helderweirt, H. V. D. Auweraer, P. Mas et al., "Application of accelero- meter-based rotational degree of freedom measurements for engine subframe modelling," in Proceedings of the 19th International Modal Analysis Conference, Orlando, FL, USA, February 2001.

[44] P. Avitabile and J. O'Callahan, "Frequency response function expansion for unmeasured translation and rotation DOFS for impedance modelling applications," Mechanical Systems and Signal Processing, vol. 17, no. 4, pp. 723-745, 2003.

[45] J. M. M. Silva, N. M. M. Maia, and A. M. R. Ribeiro, "Indirect estimation of Rotational frequency response functions," in Proceedings of the 19th International Modal Analysis Conference, Orlando, FL, USA, February 2001.

[46] D. Montalvão, A. M. R. Ribeiro, N. M. M. Maia, and J. M. M. Silva, "Estimation of the rotational terms of the dynamic response matrix," Shock and Vibration, vol. 11, Article ID 780837, 18 pages, 2004.

[47] W. Xue and H. Hua, "An overview of frequency response function synthesis based on experimental data," Noise and Vibration Control, vol. 33, no. 4, pp. 71-73, 2013.

[48] M. V. V. D. Van der Seijs, D. D. Klerk, D. J. Rixen et al., Validation of Current State Frequency Based Sub-structuring Technology for the Characterization of Steering Gear-Vehicle interaction, Springer, Berlin, Germany, 2014.

[49] D. Klerk, D. J. Rixen, S. N. Voormeeren et al., "Solving the RDoF problem in experimental dynamic substructuring," in Proceedings of the 26th International Modal Analysis Conference (IMACXXVI), Orlando, FL, USA, February 2008.

[50] P. C. Pandey and S. V. Barari, "Multilayer perecption in damage detection of bridges structures," Computers \& Structures, vol. 42, no. 4, pp. 649-659, 1992.

[51] M. J. Atalla and D. J. Inman, "On model updating using neural networks," Mechanical Systems and Signal Processing, vol. 12, no. 1, pp. 135-161, 1998.
[52] R. I. Levin, N. A. J. Lieven, and M. H. Lowenberg, "Measuring and improving neural network generalization for model updating," Journal of Sound and Vibration, vol. 238, no. 3, pp. 401-424, 2000.

[53] C. C. Chang, T. Y. P. Chang, Y. G. Xu et al., "Adaptive neural networks for model updating of structures," Smart Materials and Structures, vol. 9, no. 1, pp. 59-68, 2000.

[54] C. C. Chang, T. Y. P. Chang, Y. G. Xu, and W. M. To, "Selection of training samples for model updating using neural networks," Journal of Sound and Vibration, vol. 249, no. 5, pp. 867-883, 2002.

[55] M. W. Vanik, J. L. Beck, and S. K. Au, "Bayesian probabilistic approach to structural health monitoring," Journal of Engineering Mechanics, vol. 126, no. 7, pp. 738-745, 2000.

[56] X. Wu, J. Ghaboussi Jr., and J. H. Garrett, "Use of neural networks in detection of structural damage," Computers \& Structures, vol. 42, no. 4, pp. 649-659, 1992.

[57] Y. Lu and Z. Tu, "A two-level neural network approach for dynamic FE model updating including damping," Journal of Sound and Vibration, vol. 275, no. 3-5, pp. 931-952, 2004.

[58] P. Tsou and M.-H. H. Shen, "Structural damage detection and identification using neural networks," AIAA Journal, vol. 32, no. 1, pp. 176-183, 1994.

[59] C.-B. Yun and E. Y. Bahng, "Substructural identification using neural networks," Computers \& Structures, vol. 77, no. 1, pp. 41-52, 2000.

[60] B. Xu, Z. Wu, G. Chen, and K. Yokoyama, "Direct identification of structural parameters from dynamic responses with neural networks," Engineering Applications of Artificial Intelligence, vol. 17, no. 8, pp. 931-943, 2004.

[61] R. L. Fox and M. P. Kapoor, "Rates of change of eigenvalues and eigenvectors," AIAA Journal, vol. 6, no. 12, pp. $2423-$ 2428, 1968.

[62] L. C. Rogers, "Derivatives of eigenvalues and eigenvectors," AIAA Journal, vol. 8, no. 5, pp. 944-945, 1970.

[63] S. Garg, "Derivatives of eigensolutions for a general matrix," AIAA Journal, vol. 11, no. 8, pp. 1191-1194, 1973.

[64] R. B. Nelson, "Simplified calculation of eigenvector derivatives," AIAA Journal, vol. 14, no. 9, pp. 1201-1205, 1976.

[65] K. B. Lim, J. L. Junkins, and B. P. Wang, "Re-examination of eigenvector derivatives," Journal of Guidance, Control, and Dynamics, vol. 10, no. 6, pp. 581-587, 1987.

[66] Q. W. Zhang, C. C. Chang, and T. Y. P. Chang, "Finite element model updating for structures with parametric constraints," Earthquake Engineering \& Structural Dynamics, vol. 29, no. 7, pp. 927-944, 2000.

[67] C. Farhat and F. M. Hemez, "Updating finite element dynamic Models using an element-by-element sensitivity methodology," AIAA Journal, vol. 31, no. 9, pp. 1702-1711, 1993.

[68] F. Hemez and G. Brown, "Improving Structural dynamics models by correlating simulated to measured frequency response functions," in Proceedings of the Aiaa/asme/ascel ahs/asc Structures, Structural Dynamics, and Materials Conference and Exhibit, Long Beach, CA, USA, April 2013.

[69] S.-L. J. Hu, H. Li, and S. Wang, "Cross-model cross-mode method for model updating," Mechanical Systems and Signal Processing, vol. 21, no. 4, pp. 1690-1703, 2007.

[70] H. G. Natke, "Die Korrektur des Rechenmodells eines elastomechanischen systems mittels gemessener erzwungener Schwingungen," Ingenieur-Archiv, vol. 46, no. 3, pp. 169-184, 1977.

[71] C. D. Foster and J. E. Mottershead, "A method for improving finite element models by using experimental data: 
application and implications for vibration monitoring," International Journal of Mechanical Sciences, vol. 32, no. 3, pp. 191-203, 1990.

[72] M. Link, "Identification and correction of errors in analytical models using test data: the pretical and practical bounds," in Proceedings of the 8th International Modal Analysis Conference, pp. 570-578, Kissimmee, FL, USA, February 1990.

[73] M. I. Friswell and J. E. T. Penny, "Updating model parameters from frequency domain data via reduced order models," Mechanical Systems and Signal Processing, vol. 4, no. 5, pp. 377-391, 1990.

[74] P. O. Larsson and P. Sas, "Model updating based on force vibrations," in Proceedings of the 4th Conference on Recent Advances in Structural Dynamics, pp. 95-98, Southampton, UK, July 1991.

[75] R. M. Lin and D. J. Ewins, "Model updating using FRF data," in Proceedings of the 15th International Seminar on Modal Analysis, pp. 141-162, Leuven, Belgium, September 1990.

[76] W. J. Visser and M. A. Imregun, "Technique to update finite element models using frequency response data," in Proceedings of the 9th International Modal Analysis Conference, Florence, L. W. Alfred, J. Dominick, and DeMichele, W. J. Visser, and D. J. Ewins, "Finite element model updating using frequency response function data-I: theory and initial investigation," Mechanical Systems and Signal Processing, vol. 9, no. 2, pp. 187-202, 1995.

[78] M. Link and L. Zhang, "Experience with different procedures for updating structural parameter of analytical models using test data," in Proceedings of the 10th International Modal Analysis Conference, San Diego, CA, USA, February 1992.

[79] C. Zang, H. Grafe, and M. Imregun, "Frequency-domain criteria for correlating and updating dynamic finite element models," Mechanical Systems and Signal Processing, vol. 15, no. 1, pp. 139-155, 2001.

[80] K.-S. Kwon and R.-M. Lin, "Frequency selection method for FRF-based model updating," Journal of Sound and Vibration, vol. 278, no. 1-2, pp. 285-306, 2004.

[81] M. Imregun, K. Y. Sanliturk, and D. J. Ewins, "Finite element model updating using frequency response function data-II: case study on a medium-size finite element model," $M e$ chanical Systems and Signal Processing, vol. 9, no. 2, pp. 203-213, 1995.

[82] R. M. Lin and J. Zhu, "Finite element model updating using vibration test data under base excitation," Journal of Sound and Vibration, vol. 303, no. 3-5, pp. 596-613, 2007.

[83] S. Adhikari and M. I. Friswell, "Distributed parameter model updating using the Karhunen-Loève expansion," Mechanical Systems and Signal Processing, vol. 24, no. 2, pp. 326-339, 2010.

[84] P. O. Larsson, "Model updating based on forced vibrations," in Proceedings of the 10th International Conference on Recent Advances in Stmchmal Dvmnics, no. 2, pp. 968-974, London, UK, 1992.

[85] G. Steenackers and P. Guillaume, "Finite element model updating taking into account the uncertainty on the modal parameters estimates," Journal of Sound and Vibration, vol. 296, no. 5, pp. 919-934, 2006.

[86] S. V. Modak, T. K. Kundra, and B. C. Nakra, "Comparative study of model updating methods using simulated experimental data," Computers \& Structures, vol. 80, no. 5-6, pp. 437-447, 2002.

[87] A. Teughels, G. De Roeck, and J. A. K. Suykens, "Global optimization by coupled local minimizers and its application to FE model updating," Computers \& Structures, vol. 81, no. 24-25, pp. 2337-2351, 2003.
[88] C. Mares and C. Surace, "An application of genetic algorithms to identify damage in elastic structures," Journal of Sound and Vibration, vol. 195, no. 2, pp. 195-215, 1996.

[89] M. I. Friswell, J. E. T. Penny, and S. D. Garvey, “A combined genetic and eigensensitivity algorithm for the location of damage in structures," Computers \& Structures, vol. 69, no. 5, pp. 547-556, 1998.

[90] S. A. Dunn, "The use of genetic algorithms and stochastic hill-climbing in dynamic finite element model identification," Computers \& Structures, vol. 66, no. 4, pp. 489-497, 1998.

[91] D. C. Zimmerman, K. Yap, and T. Hasselman, "Evolutionary approach for model refinement," Mechanical Systems and Signal Processing, vol. 13, no. 4, pp. 609-625, 1999.

[92] R. I. Levin and N. A. J. Lieven, "Dynamic finite element model updating using simulated annealing and genetic algorithms," Mechanical Systems and Signal Processing, vol. 12, no. 1, pp. 91-120, 1998.

[93] J.-H. Chou and J. Ghaboussi, "Genetic algorithm in structural damage detection," Computers \& Structures, vol. 79, no. 14, pp. 1335-1353, 2001.

[94] M. A. Rao, J. Srinivas, and B. S. N. Murthy, "Damage detection in vibrating bodies using genetic algorithms," Computers \& Structures, vol. 82, no. 11-12, pp. 963-968, 2004.

[95] F. T. K. Au, Y. S. Cheng, L. G. Tham, and Z. Z. Bai, "Structural damage detection based on a micro-genetic algorithm using incomplete and noisy modal test data," Journal of Sound and Vibration, vol. 259, no. 5, pp. 10811094, 2003.

[96] A. Berman and E. J. Nagy, "Improvement of a large analytical model using test data," AIAA Journal, vol. 21, no. 8, pp. 1168-1173, 1983.

[97] Y. Halevi and I. Bucher, "Model updating via weighted reference basis with connectivity constraints," Journal of Sound and Vibration, vol. 265, no. 3, pp. 561-581, 2003.

[98] S. Wu and G. Cai, "FRF based substructuring technique considering rotational degrees of freedom of interface," Journal of Vibration Engineering, vol. 24, no. 3, pp. 323-326, 2011.

[99] A. Drozg, G. Čepon, and M. Boltežar, "Full-degrees-offreedom frequency based substructuring," Mechanical Systems and Signal Processing, vol. 98, no. 1, pp. 570-579, 2018.

[100] Y. O. U. Caixia, Z. H. A. N. G. Guangde, H. E. Xuesong et al., "The calculation method of the isolation system based on the improved FBS theory," Chinese Journal of Ship Research, vol. 10, no. 1, pp. 109-113, 2015.

[101] G. Canbaloğlu and H. Nevzat Özgüven, "Model updating of nonlinear structures from measured FRFs," Mechanical Systems and Signal Processing, vol. 80, no. 5, pp. 282-301, 2016.

[102] J. T. Wang, C. J. Wang, and J. P. ZHAO, "Frequency response function-based model updating using Kriging model," Mechanical Systems and Signal Processing, vol. 87, no. 3, pp. 218-228, 2017.

[103] L. Wang and Z. Niu, "Study of non-uniform beam model updating method based on genetic algorithm," Missiles and Space Vehicles, vol. 2, pp. 60-63, 2013.

[104] C. Gagliano, "A hybrid full vehicle model for structure borne road noise prediction," in Proceedings of the SAE 2005 noise and vibration conference and exhibition, Michigan:Society of Automotive Engineers, Traverse City, MI, USA, May 2005.

[105] J. E. Mottershead, M. Link, and M. I. Friswell, "The sensitivity method in finite element model updating," Mechanical 
Systems and Signal Processing, vol. 25, no. 7, pp. 2275-2296, 2011.

[106] K.-S. Kim and Y. J. Kang, "Local stiffness control for reducing vehicle interior noise by using FRF-based synthesis method," Journal of Mechanical Science and Technology, vol. 25, no. 1, pp. 81-88, 2011.

[107] T. Sakai, "Development procedure for interior noise performance by virtual vehicle refinement, combining experimental and numerical component models," in Proceedings of the SAE 2001 Noise and Vibration Conference and Exhibition, pp. 283-284, Michigan:Society of Automotive Engineers, Traverse City, MI, USA, 2001. 